\title{
Symmetry of Oculomotor Burst Neuron Coordinates About Listing's Plane
}

\author{
J. D. CRAWFORD AND T. VILIS \\ Departments of Physiology and Ophthalmology, University of Western Ontario, London, Ontario N6A 5C1, Canada
}

\section{SUMMARY AND CONCLUSIONS}

1. The purpose of this investigation was to determine the axes of eye rotation generated by oculomotor burst neuron populations and the coordinate system that they collectively define. In particular, we asked if such coordinates might be related to constraints in the emergent behavior, i.e., Listing's law for saccades.

2. The mesencephalic rostral interstitial nucleus of the medial longitudinal fasciculus (riMLF) was identified in four monkeys with the use of single-unit recording, and then cxplored with the use of electrical microstimulation and pharmacological inactivation with the inhibitory $\gamma$-aminobutyric acid (GABA) agonist muscimol. Three-dimensional (3-D) eye positions and velocities were recorded in one or both eyes while alert animals made eye movements in response to visual stimuli and head rotation.

3. Unilateral stimulation of the riMLF $(20 \mu \mathrm{A}, 200 \mathrm{~Hz}, 300-$ $600 \mathrm{~ms}$ ) produced conjugate, constant velocity eye rotations, which then stopped abruptly and held their final positions. This is expected if the riMLF produces phasic signals upstream from the oculomotor integrator.

4. Units that burst before upward or downward saccades were recorded intermingled in each side of the riMLF. Unilateral stimulation of the same riMLF sites produced eye rotations about primarily torsional axes, clockwise ( CW ) during right riMI F stimulation and counterclockwise (CCW) during left stimulation. Only small and inconsistent vertical components were observed, supporting the view that the riMLF carries intermingled up and down signals.

5. The torsional axes of eye rotation produced by riMLF stimulation did not correlate to external anatomic landmarks. Instead, stimulation axes from both riMLF sides aligned with the primary gaze direction orthogonal to Listing's plane of eye positions recorded during saccades.

6. Injection of muscimol into one side of the riMLF produced a conjugate deficit in saccades and quick phases, including a $50 \%$ reduction in all vertical velocities and complete loss of one torsional direction. $\mathrm{CW}$ was lost after right riMLF inactivation, and CCW was lost after left inactivation.

7. The plane that separated the intact torsional axes from the missing axes correlated with the orientation of Listing's plane. Thus, during left or right riMLF inactivation, the vertical axes of intact horizontal saccades were abnormally aligned with Listing's plane. The orientation of these axes was not correlated with external anatomic landmarks.

8. As suggested by their alignment with Listing's plane, the intact vertical axes of horizontal saccades following riMLF inactivation were orthogonal to torsional riMLF stimulation axes.

9. When trained animals attempted to saccade upward or downward between vertically displaced targets during unilateral riMLF inactivation, they would presumably do so with one of the burst neuron populations of the opposite, intact side. The observed saccades suggested that the two right riMLF populations generate $\mathrm{CCW}$-upward-rightward and $\mathrm{CCW}$-downward-leftward rotations, whereas the left riMLF generates $\mathrm{CW}$-upward-leftward and $\mathrm{CW}$-downward-rightward rotations. As this would suggest, during unilateral riMLF inactivation, oblique saccades were more affected along onc diagonal than the other, whercas horizontal saccades had reduced velocities and inappropriate vertical components.

10. As expected from the abnormal torsional velocities following unilateral muscimol injection, vertical saccades drove the eye up to $25^{\circ}$ out of Listing's plane in the direction of intact torsional velocity. The changes in axes reported above did not appear to be mechanical effects, because vestibuloocular reflex slow phase axes were not affected by the abnormal torsion.

11. We conclude that riMLF burst neuron populations control coordinate axes for vertical and torsional eye rotation as do the ocular motoneurons and their vestibular inputs, but they also control specific horizontal rotations. This particular nonorthogonal coordinate system gives the riMLF the potential to generate eye rotations about any $3-\mathrm{D}$ axis with only four neuron populations. Furthermore, burst neuron coordinates appear to be symmetrical about Listing's plane rather than external anatomic landmarks. This suggests that populations of oculomotor and skeletomotor neurons may sometimes utilize coordinate systems related to constraints in the behavior that they produce.

\section{INTRODUCTION}

Oculomotor short-lead burst neurons provide an excellent experimental model for examining the nature of brain coordinate systems. Burst neurons, which produce the phasic command for conjugate rapid eye movements, are named for the brief burst of action potentials that they produce before and during movements in a preferred direction (Fuchs et al. 1985; Luschei and Fuchs 1972; Sparks and Mays 1990). For such phasic movement commands, coordinates are essentially a set of one-dimensional movement directions ( the directions generated by individual burst neurons) that can bc combincd to express a movement in any given direction. To determine the overall movement, the magnitudes of movement along each coordinate direction must be specified as components (by discharge ratc of various burst neurons). Thus components are only meaningful if the associated coordinates are understood. Moreover, one must understand the frame of reference in which these coordinates are imbedded, such as the head, the body, or gravity fields. Recent theoretical models and behavioral experiments have frequently emphasized the importance of neural coordinate systems and their reference frames ( Hollerbach and Atkeson 1987; Pellionisz and Peterson 1988; Raphan and Cohen 1986; Robinson 1985; Simpson and Graf 1985; Soechting and Flanders 1991), but there is less direct experimental data (Anderson et al. 1985; Mays and 
Sparks 1980; Peterson and Baker 1991; Simpson et al. 1989). The purpose of the present study was to determine the coordinate directions utilized by oculomotor burst neurons, and the reference frame with which they align.

Short-lead burst neurons are segregated anatomically into populations of cells with similar directional tuning (King and Fuchs 1979; Luschei and Fuchs 1972). Thus determination of burst neuron coordinates may be simplified to the determination of axes controlled by burst populations. These populations appear to be organized like the upstream semicircular canals and downstream extraocular muscles, providing an ongoing source of debate on the impact of sensory and mechanical plant anatomy on the intervening neural coordinates (Büttner et al. 1977; Robinson and Zee 1981). Burst neurons are mainly located in two brain stem nuclei: 1 ) the paramedian pontine reticular formation (PPRF), which possesses populations for leftward and rightward movements, similar to the horizontal muscles and canals (Cohen et al. 1968; Cohen and Komatsuzaki 1972; Keller 1974; Luschei and Fuchs 1972; Strassman et al. 1986; Van Gisbergen et al. 1981 ); and 2) the mesencephalic rostral interstitial nucleus of the medial longitudinal fasciculus (riMLF), each lateral riMLF side possessing a population for upward movements intermingled with another population for downward movements (Büttner et al. 1977; Büttner-Ennever and Büttner 1978; King and Fuchs 1979; Kompf et al. 1979). Recent single-unit recordings and pharmacological inactivations of the monkey riMLF suggest that this nucleus is organized like the vertical semicircular canals and eye muscles; i.e., the vertical burst neurons in the right riMLF also produce clockwise $(\mathrm{CW})$ eye rotation, whereas the left riMLF neurons produce counterclockwise (CCW) rotations (Henn et al. 1989; Hepp et al. 1988; Vilis et al. 1989). However, these experiments did not distinguish between canal or muscle alignment.

Why has this question not been resolved? Single-unit recording has provided most of our knowledge of burst neuron physiology but is not suited to the precise determination of coordinates. The movement directions that correlate best to unit activity can deviate from the directions of rotation that are generated by neurons (Pellionisz and Peterson 1988). Furthermore, individual units may show variations unrelated to the overall behavior produced by the population, as demonstrated clearly by neural network models (Anastasio and Robinson 1990). Another problem is that burst neurons are generally thought to encode eye velocity (Robinson 1975; Tweed and Vilis 1990a), i.e., three-dimensional (3-D) axes of eye rotation, but previously such axes were not correctly recorded. We attempted to avoid these pitfalls by electrically microstimulating and pharmacologically inactivating various riMLF burst neuron populations while using a new, precise technique for measurement of instantaneous 3-D eye velocity (Tweed et al. 1990).

In contrast to the multiple classes of oculomotor-related activity found in the PPRF (Raybourn and Keller 1977), the riMLF appears to be almost exclusively comprised of short-lead burst neurons (Büttner et al. 1977; King and Fuchs 1979). Our preliminary task was to corroborate the accepted theory that the riMLF produces saccadic velocity signals, upstream from the neural "integrator" that gener- ates tonic eye position signals (Fuchs et al. 1985; Robinson 1975 ). If so, then a constant stimulus input should result in a ramplike change in eye position that holds at the end of stimulation. Eye movements of various directions, including torsion, have been observed previously during midbrain stimulation (Hassler 1972; Kompf et al. 1979; Westheimer and Blair 1975).

The first step in determining coordinates is to establish the directions of eye rotation controlled by individual riMLF burst neuron populations. The behaviorally measured canal/muscle-like scheme described above differs from a more recent scheme based on the anatomic projections of functionally identified riMLF burst neurons (Moschovakis et al. 1991a,b). Downward burst neuron projections to motoneurons were consistent with the canal/muscle scheme, but the projections of upward burst neurons suggested that they do not produce torsion. The behaviorally and anatomically determined schemes agree in predicting that stimulation of the right riMLF should produce $\mathrm{CW}$ eye rotations and left stimulation should produce $\mathrm{CCW}$ rotations about a head-fixed axis roughly orthogonal to the face. Both schemes also predict that CW saccade/ quick phase components will be abolished after right inactivation, and $\mathrm{CCW}$ rotations lost after left inactivation. However, the anatomic evidence (Moschovakis et al. 1991a) suggests that, during unilateral riMLF inactivation, upward saccades will be relatively normal, whereas the behavioral evidence predicts that both upward and downward saccades will be made with equal amounts of abnormal torsion (Vilis et al. 1989).

Neither scheme explains the suggestion of early singleunit investigations that riMLF neurons may also carry a small horizontal component (Büttner et al. 1977), or the anatomic projections that have been observed from the riMLF to the abducens nucleus (Büttner-Ennever and Büttner 1988). These observations suggest that the coordinate axes defined by the riMLF burst populations may also have a horizontal component. If so, then inactivation of the riMLF should also affect horizontal saccades.

Finally, we will examine the reference frame for burst neuron coordinates. Theoretical and experimental observations agree that the reference frame should be the head (Büttner et al. 1977; Pellionisz 1986; Robinson and Zee 1981), but to determine precise orientation within the head, direct observations are required. Unilateral stimulation of the riMLF should reveal the torsional coordinate axis, whereas the intact vertical axis following riMLF inactivation should reveal the coordinate specified by the remaining PPRF burst region. These axes might align with sensory canal coordinates (Robinson and Zee 1981), downstream muscle coordinates (Büttner et al. 1977), or something intermediate. Another possibility is that such motor coordinate systems do not align best with associated anatomic structures, but rather with some parameter of the behavior produced. It is the behavioral synergy called Listing's law, which determines the axis of rotation for a saccadic gaze shift, and thus the required ratio of activity between the various burst neuron populations (Hepp 1990; Tweed and Vilis 1990a,b; Westheimer and Blair 1972). Listing's law allows only those eye positions that can be reached from primary position by rotation about an axis in 
Listing's plane (von Helmholtz 1925; Tweed and Vilis $1990 \mathrm{~b}$ ). Might the coordinate axes controlled by oculomotor burst neuron populations be related to Listing's plane and its perpendicular primary gaze direction?

\section{METHODS}

\section{Measurement of 3-D eye positions and velocities}

Four Macaca fascicularis (coded MAR, BAR, CAS, ART) were prepared for chronic behavioral experiments, each monkey undergoing surgery under aseptic conditions and pentobarbital sodium anesthesia. During surgery a skull cap composed of dental acrylic was fastened to the animal's head, and two enameled copper search coils of $5 \mathrm{~mm}$ diam were implanted in one eye for measurement of 3-D eye position. Both coils were positioned nasally, one inferior and one superior. The method used did not require that the coils be aligned orthogonally to each other ( Tweed et al. 1990). The leads were extended temporally beneath the conjunctiva and then subcutaneously to sockets secured on the cap. In two of the animals ( $C A S$ and $A R T$ ), coils were implanted in both cyes. During experiments, the head of the alert monkey was immobilized by fixing the skull cap near the center of three orthogonal magnetic fields. These fields were in phase but operated at different frequencies $(62.5,125$, and $250 \mathrm{kHz})$. The resultant coil signals were digitized by a computer at a sampling frequency of $100 \mathrm{~Hz}$ during random eye movements, $500 \mathrm{~Hz}$ during brain stimulation, and $1,000 \mathrm{~Hz}$ during trained goal-directed saccades.

The computer was used to convert coil signals into eye position quaternions, both on-line and after experiments, by the use of a method described previously (Tweed et al. 1990). Quaternions were used because, unlike raw coil signals, they provide an accurate and simple measure of 3 -D eye position over a $360^{\circ}$ range. Quaternions represent each eye position as a fixed-axis rotation from a reference position (Westheimer 1957). This reference position was recorded while the monkey looked in the direction of the forward-pointing magnetic field.

Quaternions are composed of a scalar part $q_{0}$, and a vector part q. It is the vector part that is used for representation of data. The vector part has components along torsional, horizontal, and vertical axes fixed relative to the head. To interpret the data, one need only understand that $q$ is parallel with the axis of eye rotation relative to reference position, and its length is proportional to the magnitude of this rotation. To be specific, a quaternion is related to the axis and magnitude of a rotation as follows

$$
\begin{aligned}
& q_{0}=\cos (\alpha / 2) \\
& \mathbf{q}=\mathbf{n} \sin (\alpha / 2)
\end{aligned}
$$

The angle $\alpha$ is the magnitude of the rotation and $\mathbf{n}$ is a 3-D unit vector parallel to the axis of rotation (Tweed and Vilis 1987). At reference position, $\alpha$ equals 0 and thus $q_{0}$ equals 1 and $q$ equals 0 .

Quaternions were also used to compute angular velocities of eye rotation. Angular velocity cannot be computed by simply differentiating coil signals, quaternions, or any other measure of position change, because $\omega$ is also dependent on current eye position (Tweed et al. 1990). The general relation between position quaternions and angular velocity $\omega$ can be stated as

$$
\omega=2 \dot{q} q^{-1}
$$

We computed velocities by using a variation of this general equation that has been described previously (Crawford and Vilis 1991).

\section{Coordinate systems for data representation}

Depending on the question examined, data are presented in field coordinates, Listing's coordinates, or anatomic coordinates. In all of these systems, torsion is defined to be rotation about an axis fixed with respect to the head, rather than rotation about the line of sight. Quaternion and velocity vectors computed from raw coil signals were in coordinates defined by the magnetic fields, i.e., the torsional, vertical, and horizontal axes were parallel with the field directions. This field coordinate system was rather arbitrary, because animals were placed within the fields at our best estimate of an upright position.

It was sometimes necessary to rotate these data into a Listing's coordinate system. By this we mean a system with vertical and horizontal coordinates aligned in Listing's plane, and the torsional coordinate parallel with the primary gaze direction. This direction, as defined originally, is orthogonal to Listing's plane (von Helmholtz 1925), which varies with respect to anatomic landmarks (Tweed and Vilis 1990). Thus primary gaze is not necessarily equivalent to a central or straight-ahead direction, contrary to popular usage of the term. Listing's law predicts that, during saccades and fixation, the quaternion vectors of eye positions (the axes for rotations that would take the eye directly from reference position to current position) will align in a plane. This plane will not necessarily be Listing's plane, unless the reference position happens to be primary position. However, by computing the orientation of the plane with respect to the gaze direction at reference position, one can determine Listing's plane and the orthogonal primary gaze direction. Quaternions and velocities are then easily rotated into this coordinate system (Tweed et al. 1990).

Data were also rotated into coordinates on the basis of headfixed anatomic structures. This required determination of the orientation of each animals stereotaxic coordinates within the field coordinate system. In the first two animals (MAR and BAR) this was done by measuring the angle between a line from the center of the external auditory canal to the orbital canthus, and the horizontal field direction. In the other animals, search coils, aligned with the stereotaxic horizontal and sagittal planes, were implanted in the skull cap.

Whatever the coordinate system, we view velocity and quaternion axes from three standard perspectives: from above the animal, from the animal's right side, and from behind the animal, depending on which axis components are most relevant. The particular perspectives used on the figures below are indicated by head caricatures.

\section{Elicitation of eye movements}

In all animals, spontaneous saccades were made to visual targets, presented in positions meant to reveal the animal's full range of attainable eye positions and saccade axes. In two animals ( CAS and $A R T$ ) this was also done while the animal's head was rotated about the torsional, vertical, and horizontal axes, as described previously (Crawford and Vilis 1991). For some parts of this study, it was necessary to know the intended direction of gaze shifts. Therefore the other two animals ( $M A R$ and $B A R$ ) were trained to saccade between known visual targets. For this, horizontal and vertical eye position signals were monitored by a computer that ran a program controlling activation of light-emitting diodes (LEDs) on a target screen $1 \mathrm{~m}$ from the monkey's face. When the monkey fixated on one LED within a spatial window, the LED turned off and a second LED turned on simultaneous to triggering of data sampling. The program provided the monkey with a drop of grape juice when it properly fixated the second LED. Control eye movements were recorded at the beginning of each experiment for comparison with movements during riMLF stimulation and inactivation. 


\section{Invasive neurophysiological procedures}

GENERAL PROCEDURES. When training was completed, the final surgery was performed, in which a stainless steel recording chamber was mounted stereotaxically over a trephine hole in the skull centered at $8 \mathrm{~mm}$ anterior and $0 \mathrm{~mm}$ lateral, directly over the oculomotor nucleus (Shantha et al. 1968). During experiments, a hydraulic microdrive attached to the recording chamber was used to advance electrodes and cannulas into the midbrain. After the midbrain oculomotor region had been located, one electrode penetration was generally made per day. The regions of oculomotor activity along this penetration were examined by the use of singleunit recording and microstimulation and pharmacological inactivation techniques. In this fashion, each animal's midbrain oculomotor region was systematically explored with an orderly grid of electrode penetrations over a period of several months.

UNIT RECORDING AND STIMULATION. Monopolar tungsten elcetrodes (Frederick Hacr, $4 \mathrm{M} \Omega$ ) were used to record from units and to stimulate. The electrode was inserted in an insulated guide cannula, which was manually advanced into the brain stem to within $5 \mathrm{~mm}$ of the selected oculomotor region. Subsequently, the electrode was hydraulically advanced by up to $10 \mathrm{~mm}$ from the guide cannula. Oculomotor units were identified by comparing their discharge patterns with eye movements and positions. Unit recording was performed while the head-stationary animal made saccades in Listing's plane, but not during torsional vestibuloocular reflex (VOR), so correlations between unit frequencies and torsional eye movements could not be made.

After units were recorded, the same electrode was used to microstimulate at 0.5 -mm-vertical intervals through oculomotor-related structures. The standard stimulus was a series of monophasic cathodal square pulses delivered at $20 \mu \mathrm{A}$ and $200 \mathrm{~Hz}$. for durations of 300-600 ms. Stimulation was delivered at random intervals while animals spontaneously saccaded to targets throughout their visual range. Mean angular velocity of an individual stimulus-evoked eye movement was computed over the longest interval that was not interrupted by saccades. This involved division of final position by initial position, a method that is correct for 3-D and accurate for slow movements (Crawford and Vilis 1991).

PHARMACOLOGICAL INACTIVATION OF OCULOMOTOR NEURONS. After completing stimulations, the electrode was pulled out of its guide tube without otherwise disturbing the tube or the microdrive. Then a 30-gauge cannula was inserted into the guide tube and lowered to a depth determined during the unit recording and stimulation phases, usually just above the peak burst region. This did not appear to affect eye movements before the subsequent injection. The injection cannula was connected to a hamilton syringe, which was used to deliver $0.3 \mu \mathrm{l}$ of $0.05 \%$ muscimol solution to the selected site. Muscimol has a powerful inhibitory effect on neurons that possess $\gamma$-aminobutyric acid (GABA) receptors. For comparison, $0.3 \mu \mathrm{l}$ of a $2 \%$ GABA solution was also injected in two animals. Eye movement recordings commenced immediately after the injection and continued for $30 \mathrm{~min}$ or more. Afterward the animal was allowed a 48 -h recovery pcriod, and then experiments were repeated at an adjacent brain site displaced lateral-medial or rostral-caudal by $1 \mathrm{~mm}$.

HISTOLOGY. When the midbrain oculomotor region had been thoroughly explored, the animals were deeply anesthetized with pentobarbital, and electrolytic lesions ( $1.5 \mathrm{~mA}$ DC anodal current for $15 \mathrm{~s}$ ) were made at a reference microdrive coordinate. Immediately afterward, animals were given a lethal dose of anesthetic and perfused with an intra-aortic injection of Formalin. The brains were removed, sliced into $100-\mu \mathrm{m}$ sections, and stained with thionin. The resulting slides were compared with a stereotaxic atlas of the monkey brain (Shantha et al. 1968) to confirm the anatomic locations of the recording sites.

\section{Identification of the riMLF burst region}

Before examining the properties of the riMLF burst neuron region, it was necessary to correctly identify this region and distinguish it from nearby oculomotor structures that are functionally distinct. Single-unit recordings identified a region in the midbrain with a high concentration of short-lead burst neurons, within stereotaxic coordinates extending from 8 to $11 \mathrm{~mm}$ anterior, 7.0 to $5.5 \mathrm{~mm}$ vertically, and laterally as far as $2.5 \mathrm{~mm}$. These neurons were classified according to their highly distinctive saccade-related activity, as described previously by several authors (Büttner et al. 1977; King and Fuchs 1979). RiMLF units produced a high-frequency burst of action potentials beginning several milliseconds before saccades in their preferred direction, the number of spikes being proportionate to the amplitude of the upward saccade component in some units, and the downward component in other units. Up-tuncd and down-tuned burst neurons were intermingled on each lateral side. This type of hurst activity was recorded at all of the riMLF stimulation and inactivation sites examined in RESULTS.

Oculomotor activity of adjacent anatomic sites was also examined. Units carrying high-frequency, machine-like burst-tonic activity similar to that of the more posterior third cranial (oculomotor) nucleus were recorded $3 \mathrm{~mm}$ below the burst region. Such units were encountered and lost within electrode excursions of several micrometers, suggesting that they were axon fibers. Stimulation of this and other motoneuron regions produced a highly distinctive type of disconjugate cyc rotation that was never ob-

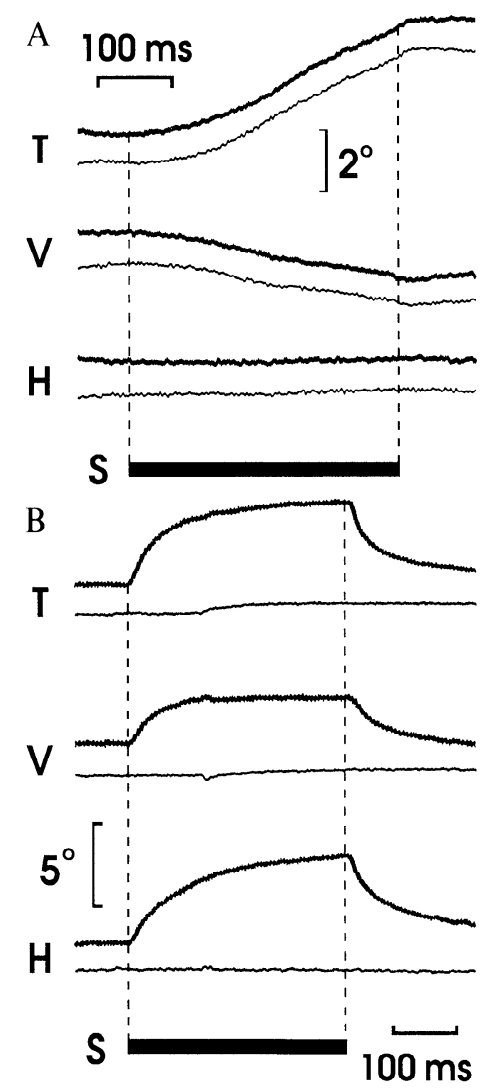

FIG. 1. Binocular eye position during unilateral microstimulation of the $(A)$ right rostral interstitial nucleus of the medial longitudinal fasciculus $(B)$ 3rd cranial nerve. Torsional ( I ), vertical ( V), and horizontal ( H) positions of the eyes are plotted as a function of time. Thick lines, left eye; thin lines, right eye. The duration of the standard stimulus $(\mathrm{S} ; 20 \mu \mathrm{A}, 200$ $\mathrm{Hz}$ ) is indicated by the bar. Data are plotted in Listing's coordinates, so that torsion is equivalent to violation of Listing's law. Animal: $A R T$ 
served during riMLF stimulation (Fig. 1). Irregular (compared with motoneurons) burst-tonic activity was recorded in a region corresponding to the interstitial nucleus of Cajal (INC), which was ventral, medial, and immediatcly caudal to the burst region. INC unit activity was correlated with vertical eye position as well as saccade velocity. The riMLF was clearly distinguishable from the INC by the pronounced vertical-torsional eye position drift that was observed immediately after inactivation of the latter structure (Crawford et al. 1991). This functional identification of the mesencephalic oculomotor structures was consistent with the known anatomy of the region, as confirmed by our histological procedures. The relative anatomic segregation of riMLF burst units suggested that they could be manipulated without affecting other oculomotor populations.

Injection of muscimol directly into the riMLF region produced immediate oculomotor deficits, whereas an injection $1 \mathrm{~mm}$ outside this identificd region required $\sim 30$ min before a prominent oculomotor deficit became apparent. This suggested that, in this time period, muscimol inactivated oculomotor units within a sphere of a diameter slightly more than $1 \mathrm{~mm}$. Injection of GABA directly into a burst neuron site produced the same initial effects reported below for muscimol, but recovery was observed within $10 \mathrm{~min}$. For brevity we will henceforth refer to injection of muscimol into one side of the riMLF (usually $2 \mathrm{~mm}$ lateral) as unilateral riMLF inactivation, without implying that every burst neuron on that side had necessarily been inactivated.

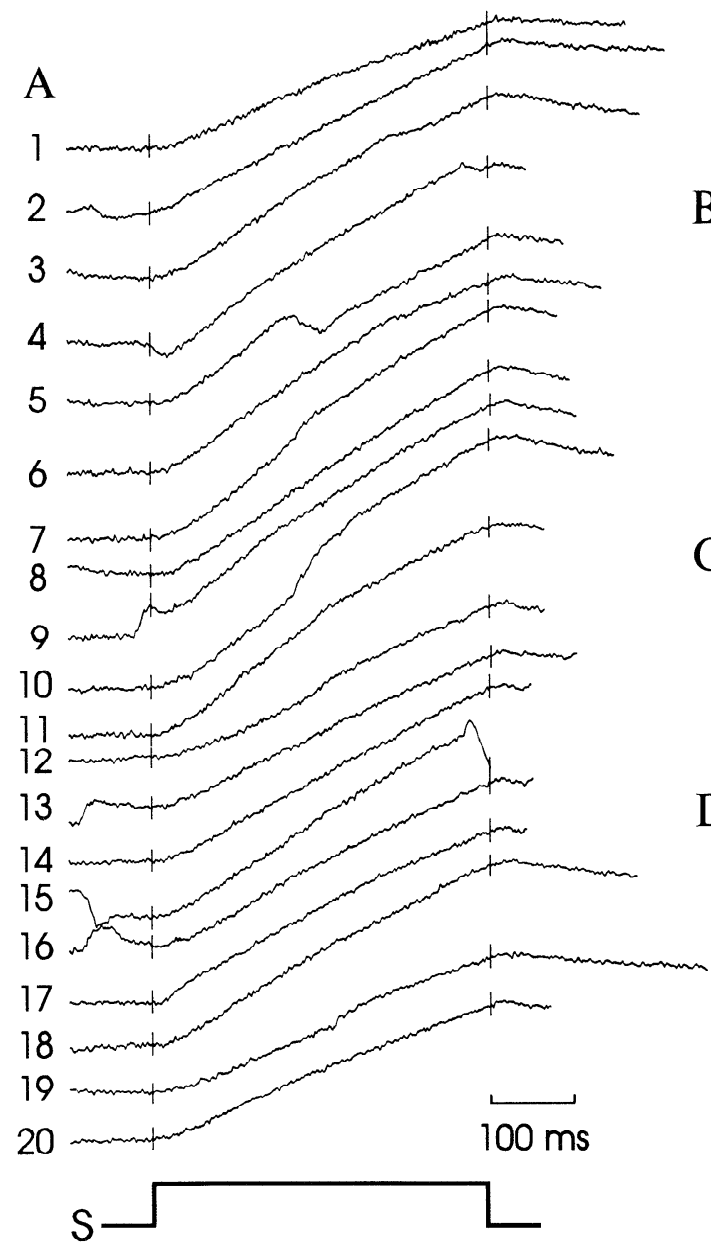

\section{RESULTS}

\section{Microstimulation of the riMLF}

Electrical microstimulation of identified burst neurons was performed at 26, 39, 13, and 17 sites in animals $M A R$, $B A R, C A S$, and $A R T$, respectively. Ten to 20 stimulations were typically delivered at each of these sites, sometimes up to 100 . The results reported below were observed consistently in all of these trials, except where specifically stated otherwise. As explained in METHODS, when burst neurons were recorded during saccades, the two sides of the riMLF appcared to be similar, each possessing intermingled upand down-tuned neurons. During unilateral riMLF stimulation, these vertical components appeared to cancel each other, and the remaining components emphasized the difference between the two sides. The primary change in eye position during stimulation was torsional, out of Listing's plane of normal saccadic eye positions. The direction of movement was $\mathrm{CW}$ during right riMLF stimulation and CCW during left riMLF stimulation. We will first consider the time course of stimulus-evoked eye position changes, and then precise directions of rotation.

TIME COURSE OF EYE POSITION CHANGE. Figure $1 A$ illustrates the conjugate nature of a typical eye movement re-
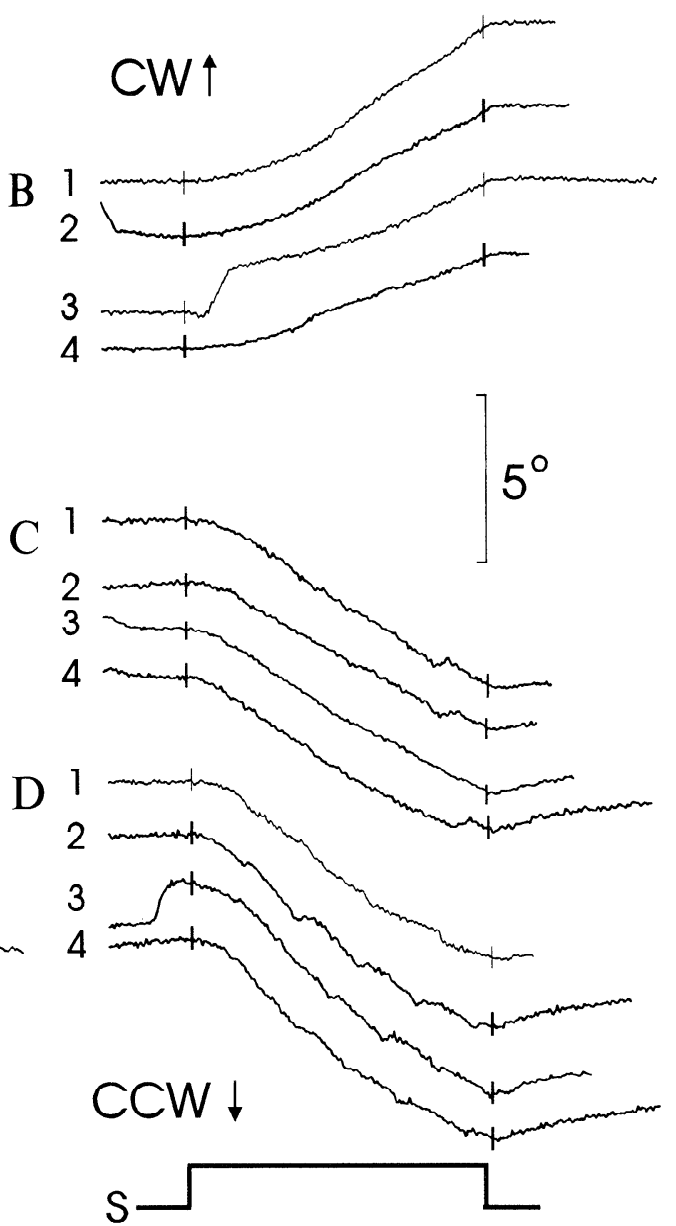

FIG. 2. Ramplike changes in torsional eye position during steplike stimulation ( $S$ ) of the rostral interstitial nucleus of the medial longitudinal fasciculus ( riMLF). Right riMLF stimulations are illustrated for animal $C A S(A)$ and animal $A R T(B)$. Left riMLF stimulations are also illustrated for $C A S(C)$ and $A R T(D)$. Stimulations were performed in the light at random gaze directions. $\mathrm{CW}$, clockwise; $\mathrm{CCW}$, counterclockwise. 
corded during right riMLF stimulation. Data are plotted in Listing's coordinates, so that torsional position is relative to Listing's plane. For contrast, eye movements evoked by stimulation of putative motoneuron fibers several millimeters below the riMLF (Fig. $1 B$ ) are also shown. During such stimulations and during stimulations of the more posterior third cranial (oculomotor) nucleus, the eye movement was not conjugate. In the illustrated case, only the ipsilateral eye moved, as would be expected from stimulation of the third cranial nerve below its decussations. Furthermore, during application of the stimulus, the eye drifted exponentially toward a new position. On termination of the stimulus, the eye drifted rapidly back toward its original position. This is the behavior expected from a step input directly to the motoneurons supplying one eye, downstream from the neural integrator.

RiMLF stimulation produced changes in cyc position at a much more constant rate than motoneuron stimulation. Plots of eye position as a function of time during applica-

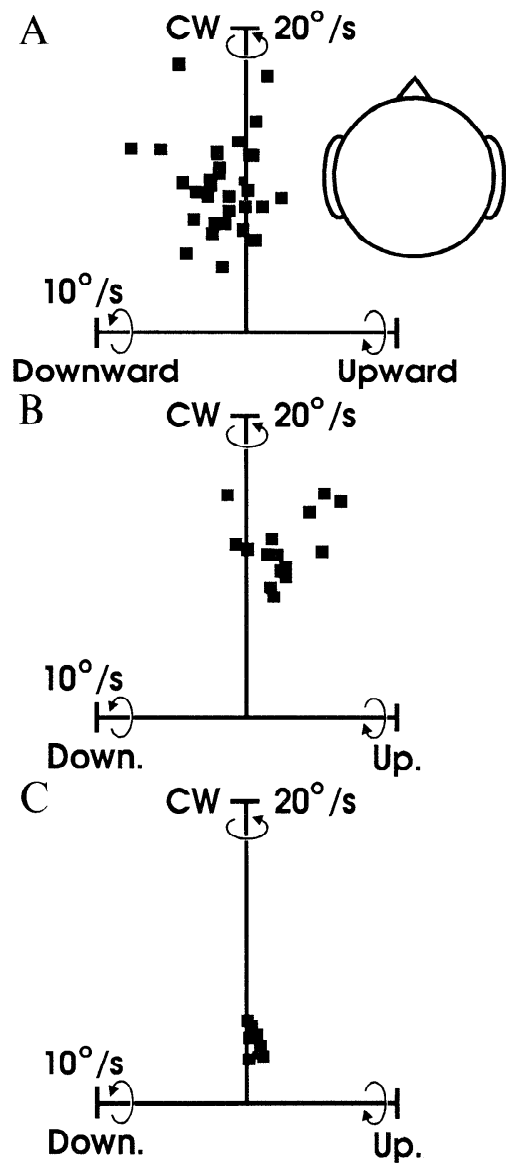

FIG. 3. Mean eye velocities during multiple stimulations at $0.5-\mathrm{mm}-$ vertical intervals along 1 right rostral interstitial nucleus of the medial longitudinal fasciculus electrode penetration in animal $A R T$. Stereotaxic coordinates: anterior, $10 \mathrm{~mm}$; lateral, $2 \mathrm{~mm}$; vertical, $(A) 7.0,(B) 6.5$, and (C) $6.0 \mathrm{~mm}$. Eye movements were not evoked $0.5 \mathrm{~mm}$ above $(A)$ or 0.5 $\mathrm{mm}$ below $(C)$. Data points are the mean velocities of individual stimulusevoked movements, plotted in field coordinates, and viewed from a perspective above the subject, as indicated by the head caricature. Stimulations were performed in the light at random gaze directions. Although care was taken not to include saccadic movements in the data, axis variability increased with the frequency of spontaneous saccades, perhaps because of variable natural neural inputs to the burst neurons during intersaccadic intervals.
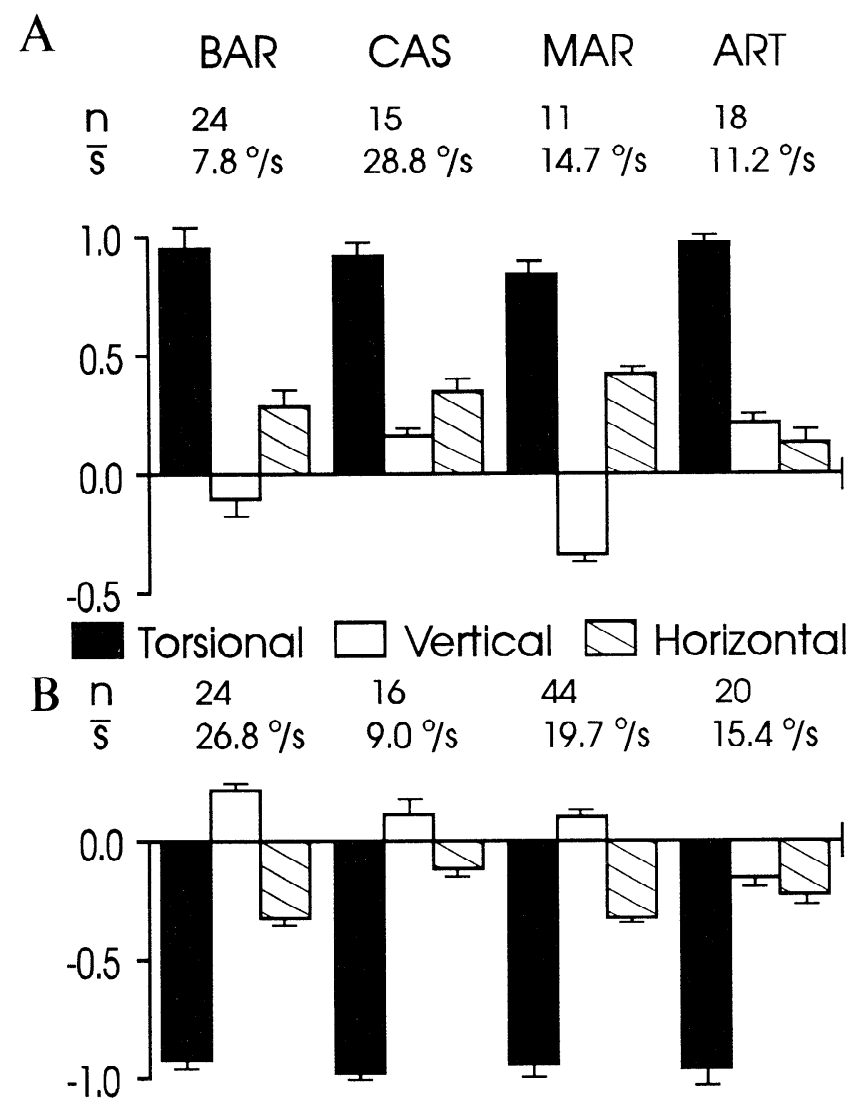

FIG. 4. Normalized components of representative eye velocities from right rostral interstitial nucleus of the medial longitudinal fasciculus (riMIF; $A$ ) and left $(B)$ riMLF of the 4 animals. Positive deflection denotes clockwise, upward, or leftward rotation. See text for explanation.

tion of the standard steplike stimulus to the riMLF resembled a ramp (Fig. 2). Another important observation was that, on termination of the stimulus, eye position stopped abruptly and held, without the rapid drift obscrved during motoneuron stimulation. The consistency of these observations is emphasized in Fig. $2 A$, which shows torsional eye position (in Listing's coordinates) as a function of time for a series of 20 consecutive right riMLF stimulations. Eye positions have been truncated at the occurrence of the first poststimulus saccade, which will not be considered here. Also shown are several right riMLF stimulations from a second animal (Fig. $2 B$ ), and several left riMLF stimulations from the same two animals (Fig. 2, $C$ and $D$ ). The latter also exhibit the typical ramplike response and stability of poststimulus eye position.

The ramplike response was most clear when no saccades occurred during the stimulus (Fig. $1 A$ ). However, the ramp was usually superimposed on horizontal and vertical saccades, which occurred frequently during the relatively long stimulus. The directions and sizes of these saccades were not consistent, and neither was their affect on the torsional ramp. For example, many saccades occurred during the movements illustrated in Fig. 2, some reducing torsion (e.g., midmovement $A 5$ ), some increasing the torsion (e.g., early in movement $B 3$ ), but more often inducing random noise as in Fig. $2 D$.

DIRECTION OF EYE ROTATION. Stimulations throughout one side of the riMLF consistently produced eye rotations that 
were mainly torsional, $\mathrm{CW}$ during right stimulation and $\mathrm{CCW}$ during left stimulation. Figure 3 illustrates the mean eye velocities produced by multiple stimulations at 0.5 $\mathrm{mm}$-vertical intervals through the right riMLF. Eye velocities are viewed from a perspective above the animal, as indicated by the head caricature, in magnetic field coordinates. A line drawn from the origin to each data point defines the axis of rotation, the length of the line is the speed, and direction of rotation is defined by the right-hand convention. If the thumb of the right hand is pointed along this line away from the origin, then the fingers curl in the direction of rotation. As the stimulation electrode was vertically advanced, the onset of eye movement was abrupt, going from no response at $0.5 \mathrm{~mm}$ above the illustrated range to a large response (Fig. 3, $A$ and $B$ ), and then becoming smaller (Fig. $3 C$ ). This transition was similar for both eyes, which always rotated with similar directions and speeds. The observed variations in eye speed and direction for any one stimulation site were not correlated with eye position. Again, the primary direction of eye rotation was torsional, $\mathrm{CW}$ for the right riMLF sites illustrated. A small tendency
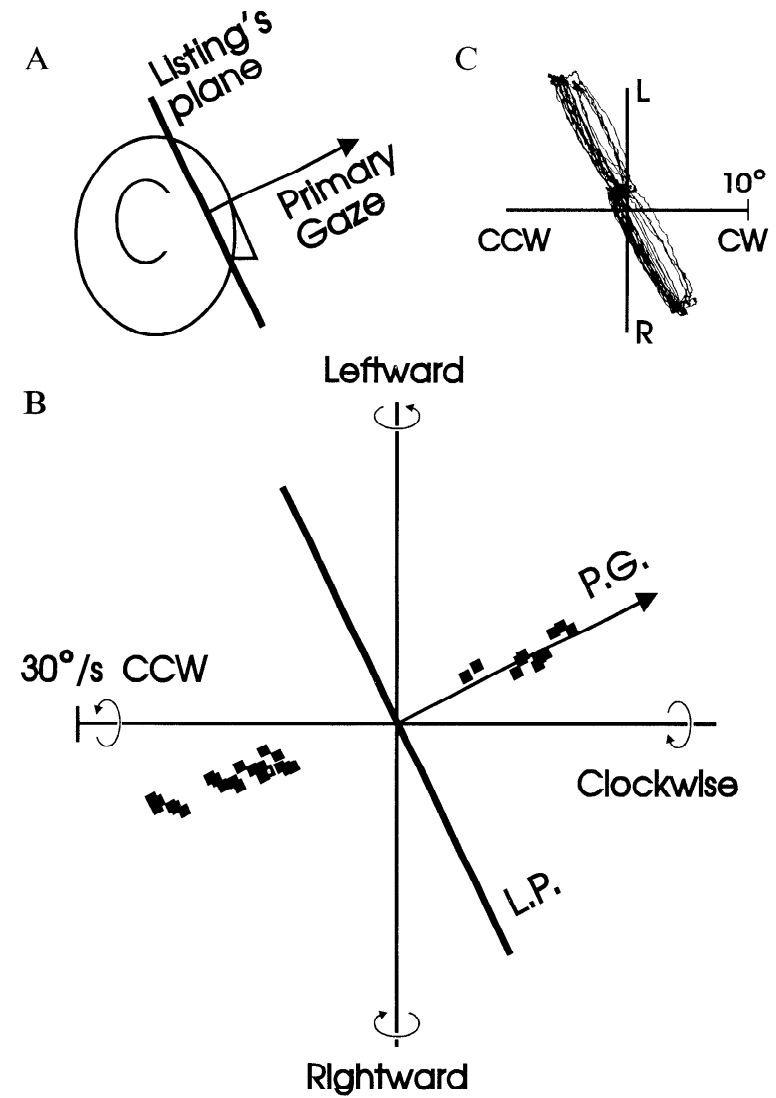

FIG. 5. Correspondence between rostral interstitial nucleus of the medial longitudinal fasciculus (riMLF) stimulation axes and primary gaze direction. $A$ : schematic drawing of upward tilt of Listing's plane and its orthogonal primary gaze direction relative to the head when viewed from the side. $B$ : a real Listing's plane, viewed edge-on from the side in field coordinates. Eye positions during 40 saccades between a central target and 4 diagonal targets are plotted relative to the computed primary position. Animal: MAR. $C$ : side views of mean eye velocities during a series of stimulations at the left (counterclockwise points) and right (clockwise points) riMLF of animal MAR. A plane of best fit (L.P.) to the data in $B$ and its orthogonal (primary gaze direction; P.G.) are overplotted. Data are plotted in field coordinates.
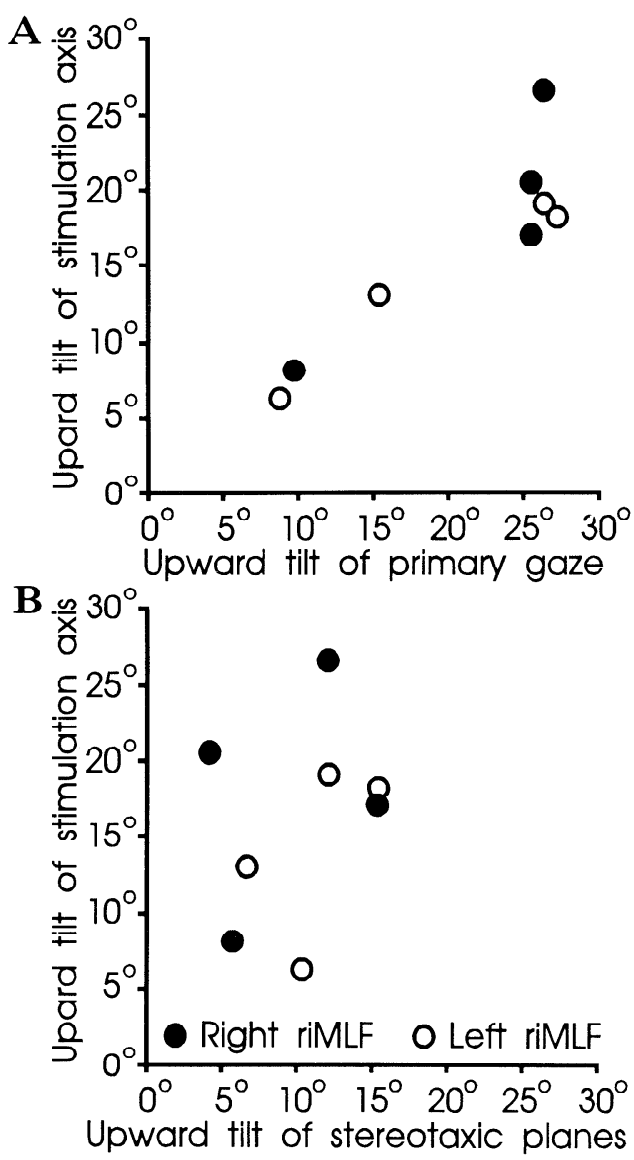

FIG. 6. Plot of upward tilt of representative stimulus axes from optimal sites of all 4 animals (Fig. 4 ) as a function of upward tilt of $(A)$ primary gaze direction (orthogonal to Listing's plane) and $(B)$ stereotaxic planes, relative to magnetic field directions.

toward vertical rotation was also observed, c.g., downward in Fig. $3 A$ and upward in Fig. $3 B$, but these directions did not appear to follow a consistent anatomic pattern. Similar results were observed during left riMLF stimulation, except that the main component of eye velocity was $\mathrm{CCW}$.

To compare data among animals, the mean stimulation velocities from each site (e.g., Fig. $3 A$ ) were averaged to get a representative stimulation velocity in field coordinates. Such representative velocities are illustrated in Fig. 4 for right riMLF and left riMLF sites of all four animals. The criterion used for choosing these sites was that they gave the largest responses of those sites removed by at least $1 \mathrm{~mm}$ anterior from areas where cell recording revealed tonic oculomotor activity and muscimol injection produced positional drift. Velocities in Fig. 4 have been normalized to unit vectors using division by average speed $(\hat{s})$. Error bars show the small standard errors of the torsional, vertical, and horizontal components. Figure 4 illustrates the main points described above, showing the dominance of the torsional component over the others, its consistency of direction $[\mathrm{CW}(+)$ for right riMLF and CCW (-) for left riMLF] and the variability of the vertical response.

Stimulation velocities plotted in field coordinates also had a consistent pattern of horizontal components (Fig. 4). Right riMLF stimulation velocities had a leftward ( + ) component, and left riMLF velocities had a rightward (-) component. If we recall that horizontal rotation occurs about a 
vertical axis, this means that the forward pointing $(\mathrm{CW})$ right riMLF stimulation vectors also pointed slightly upward, and the left riMLF stimulation vectors pointed in the opposite direction. In other words, the stimulation axes were rotated upward from the forward-pointing torsional field coordinate.

Listing's plane and its orthogonal primary gaze direction also tended to be rotated upward compared with the arbitrary field coordinate system, as indicated by the cartoon in Fig. $5 \mathrm{~A}$. That is to say that, during and between saccades, cyc position was confined to an upward-rotated plane (Fig. $5 B$ ). Figure $5 C$ shows a computer fit of Listing's plane and primary gaze direction to normal saccadic eye positions (Fig. $5 B$ ) and also the mean velocity vectors of a scrics of stimulations from the optimal right and left riMLF sites of the same animal. There was a remarkable alignment between primary gaze direction and the riMLF stimulation axes viewed from the side.

To quantify this alignment, we plotted the upward tilts of the optimal stimulation axes from Fig. 4 as a function of the upward tilt of the primary gaze direction (Fig. 61) and as a function of the upward tilt of the horizontal stereotaxic plane (Fig. $6 B$ ), each relative to the field coordinate system for a given experiment. Statistically, the upward tilt of Listing's coordinates accounted for $82 \%\left(r^{2}=0.82\right)$ of the variability in stimulation axis tilt (Fig. $6 \mathrm{~A}$ ). Furthermore, one can see that the points align closely with the line of equality. As reported previously (Tweed and Vilis 1990b), the orientation of Listing's plane varied with respect to anatomic landmarks (not illustrated). The Listing's planes used for these computations tilted upward from the stereotaxic horizontal plane by $10.4 \pm 6.9^{\circ}$ ( mean + SD), and the tilts of these two systems relative to field coordinates were not highly correlated $\left(r^{2}=0.24\right)$. The correlation between upward tilts of the stimulation axes and upward tilts of the horizontal planes (Fig. $6 B$ ) was even lower $\left(r^{2}=0.09\right)$, much lower than the correlation between stimulation axes and Listing's planes. Henceforth, data are plotted in Listing's coordinates, unless stated otherwise.

\section{Muscimol inactivation of the riMLF}

AXES OF EYE ROTATION. Muscimol was injected directly into 9, 6, 7, and 9 identified burst neuron sites in animals $M A R, B A R, C A S$, and $A R T$, respectively, as well as a greater number of immediately adjacent sites. Unilateral riMLF inactivation produced a conjugate deficit in vertical and torsional rapid eye movements, and a smaller deficit in horizontal rotations, which was observed consistently in the spontaneous saccades of all four animals. The nature of the deficit was most clcar during trained saccades between LEDs (animals MAR and BAR) and during torsional VOR quick phases (animals $C A S$ and $A R T$ ), as illustrated below.

Which axis will the eye rotate about if a trained animal attempts a vertical saccade during unilateral riMLF inactivation? Saccades between vertically displaced LED targets were normally made about a horizontal axis (Fig. $7 \mathrm{~A}$ ). This is illustrated with the use of the instantaneous velocities of these saccades, which, starting from zero, grew to a maximal value and then looped back to zero along the horizontal axis. During unilateral riMLF inactivation the velocity of saccades between vertically displaced LEDs was reduced by $50 \%$ or more (Fig. 7, top row). Furthermore, animals were unable to make purely downward or upward saccades without large torsional components. During right riMLF inactivation, both downward and upward saccades had an inappropriate $\mathrm{CCW}$ velocity component as large as the vertical component (Fig. 7B, above view). Similarly, during left inactivation, upward and downward saccades had an inappropriate $\mathrm{CW}$ velocity component as large as the vertical component (Fig. $7 C$, above view). After bilateral riMLF inactivation, vertical saccades were practically abolished (Fig. $7 D$ ).
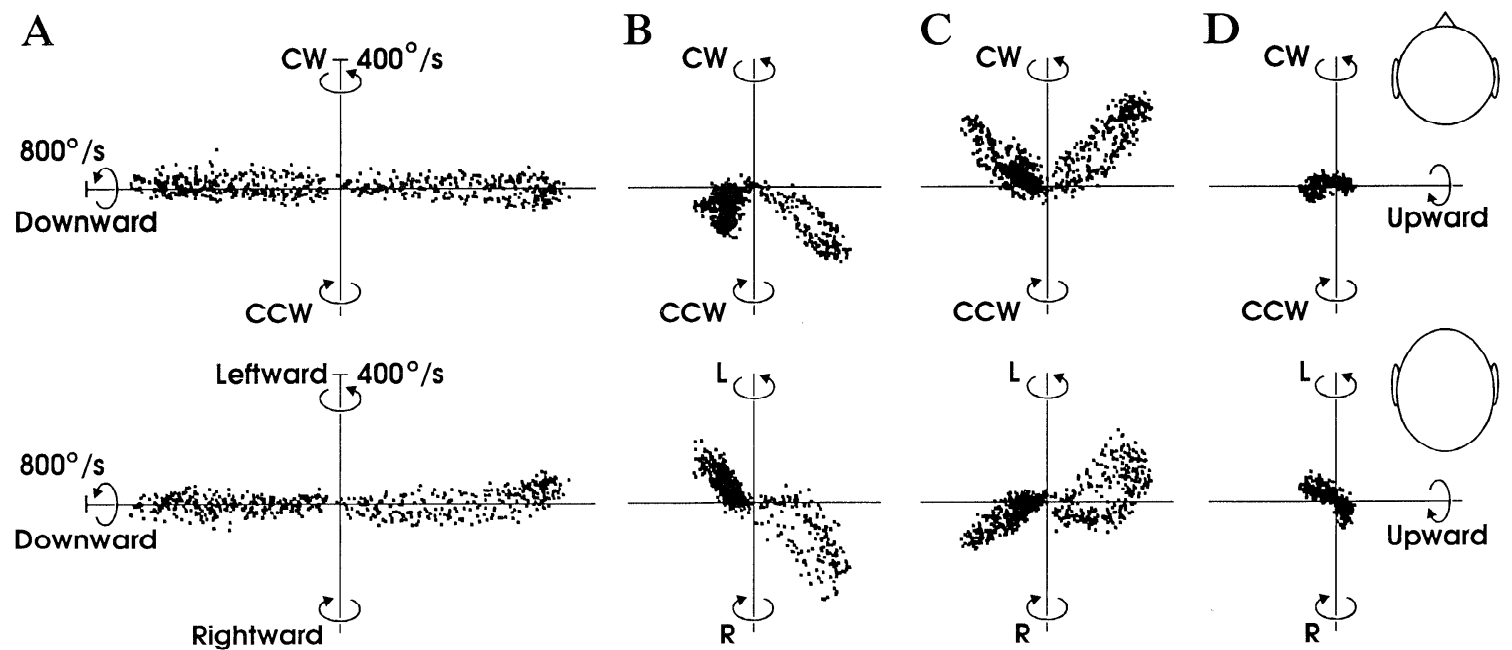

FIG. 7. Instantaneous velocities of saccades that attempt to shift gaze between targets displaced vertically by $20^{\circ},(4)$ normally, $(B)$ after right rostral interstitial nucleus of the medial longitudinal fasciculus (riMLF) inactivation, $(C)$ after left riMLF inactivation, and $(D)$ after bilateral inactivation. For each saccade, velocity begins at 0 and then loops out and back along the axis of rotation. Overlapping velocity loops of 8 consecutive upward and downward saccades are shown in each case, constituting four individual data files. Data are viewed from a perspective above (top row), and behind (bottom row) the animal, in Listing's coordinates. Animal: MAR. 


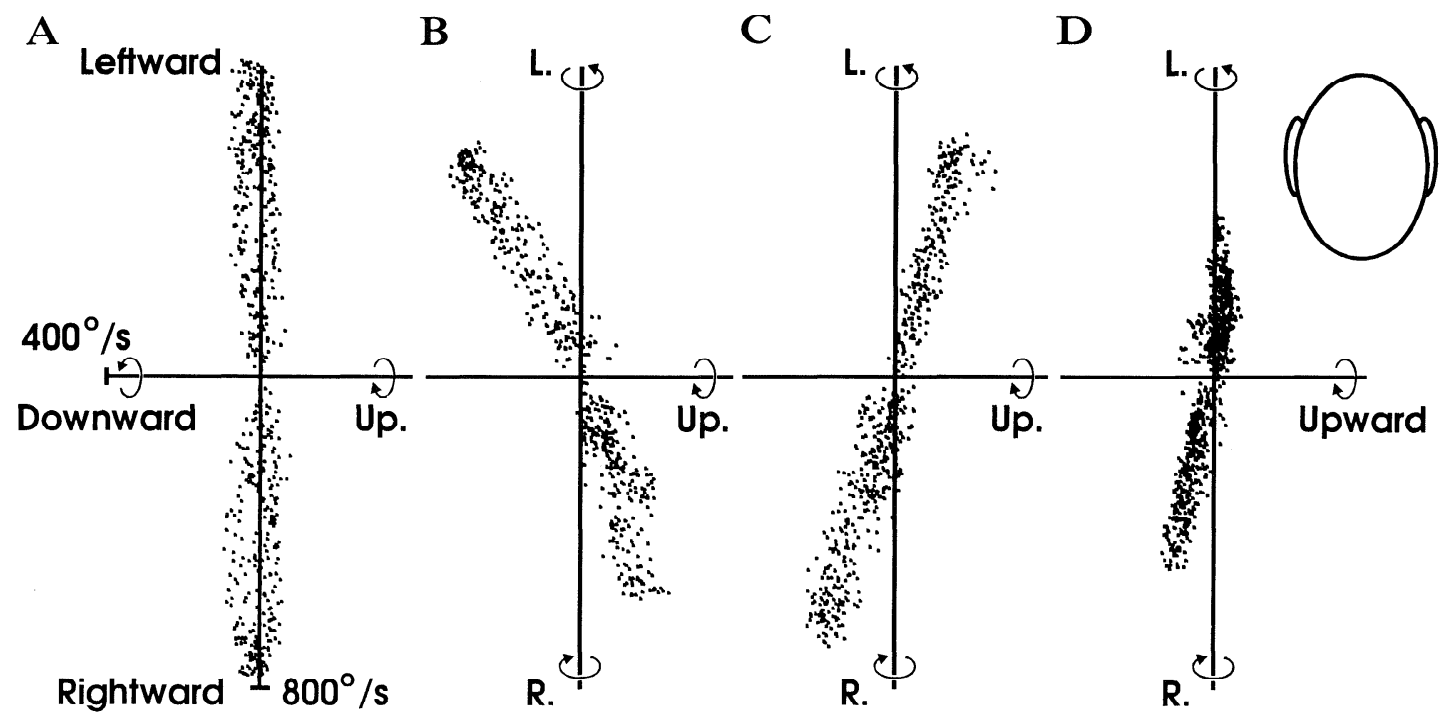

FIG. 8. Velocities of saccades that attempt to shift gaze between horizontally displaced targets, normally $(A)$ and after $(B)$ right rostral interstitial nucleus of the medial longitudinal fasciculus (riMLF) inactivation, left riMLF inactivation $(C$ ), and after bilateral inactivation $(D)$. Each panel displays overlapping velocities of 8 leftward and rightward saccades viewed from a bchind perspective in Listing's coordinates.

When these same saccade velocities were examined from a perspective behind the subject, i.e., vertical versus horizontal velocity, a surprising linkage between vertical and horizontal velocity was observed (Fig. 7, bottom row). During right riMLF inactivation, upward saccades rotated the eye rightward, and downward saccades rotated the eye leftward (Fig. $7 B$, behind view). During left riMLF inactivation the symmetrically opposite vertical-horizontal linkage was observed (Fig. $7 C$, behind view). These horizontal components were observed consistently but were usually smaller than the torsional and vertical components. Thus, when the two trained animals attempted vertical saccades during right riMLF inactivation, $\mathrm{CCW}$-upward-rightward and CCW-downward-leftward rotations occurred, and during

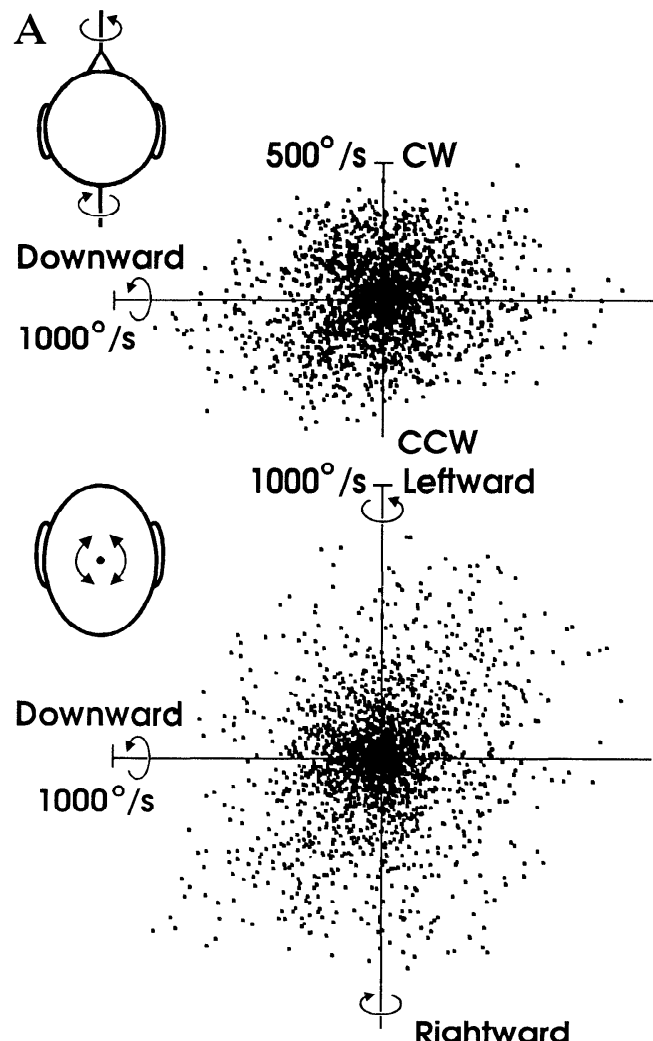

B Left Eye Right Eye
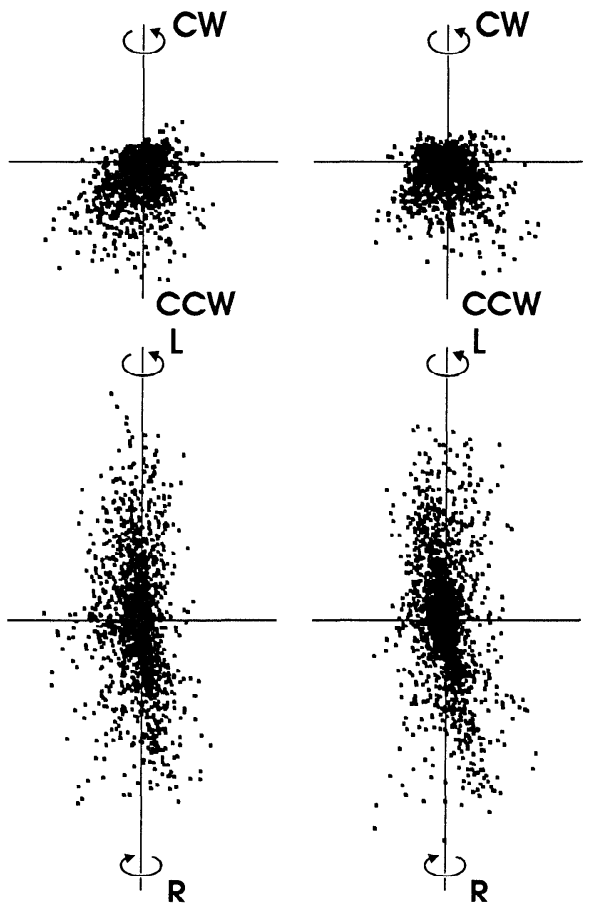

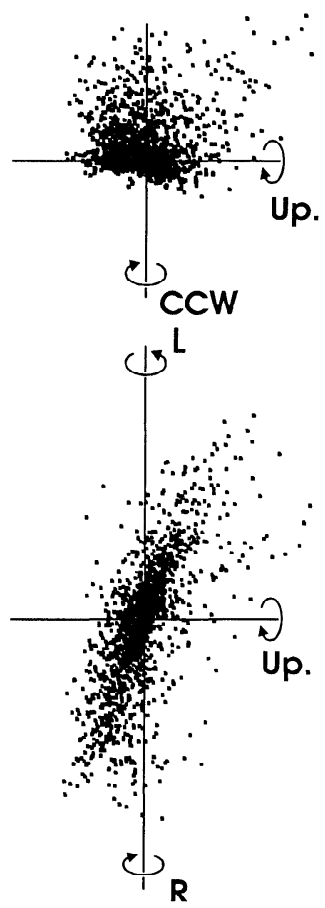

FIG. 9. Instantaneous velocities of rapid eye movements during $100 \mathrm{~s}$ of torsional vestibuloocular reflex, normally $(A)$, 22 min after injection of muscimol into the right rostral interstitial nucleus of the medial longitudinal fasciculus ( riMLF; $B$ ), and 14 min after left riMLF injection $(C)$. Data are plotted in Listing's coordinates from the above view (top row) and behind view ( bottom row). Cartoons indicate both the perspective of view and the axis of head rotation. Animal: $A R T$. 
left riMLF inactivation CW-upward-leftward and CWdownward-rightward rotations occurred.

One interpretation of the above data is that the axes used for vertical saccades during unilateral riMLF inactivation are the coordinate axes controlled by the upward and downward burst neuron populations of the opposite intact side. This would suggest that these burst populations are not only involved in generating vertical and torsional saccade components, but also horizontal components. If so, then riMLF inactivation should reduce horizontal saccade velocity. Furthermore, the riMLF would normally have to participate in horizontal saccades in such a way that its vertical components would cancel, and these should no longer cancel during unilateral inactivation. The predicted pattern was consistently observed when the two trained animals saccaded between horizontally displaced LEDs (Fig. 8 ). During right riMLF inactivation, rightward saccades had an inappropriate upward component, and leftward saccades had an inappropriate downward component (Fig. $8 B$ ). The symmetrical pattern was observed during left riMLF inactivation (Fig. $8 C$ ). Horizontal velocity was slightly reduced by unilateral riMLF inactivation (Fig. 8, $B$ and $C$ ), and further reduced by bilateral inactivation (Fig. $8 D)$.

We examined this further in the other two animals ( $C A S$ and $A R T$ ) by cliciting the maximal possible rangc of velocities before and after muscimol injection. To elicit the maximal range of movement, the monkey was rotated sinusoidally about a torsional axis, which elicited large torsional quick phases. At the same time, visual targets were presented to encourage large movements in all combinations of vertical and horizontal directions. Normally, the range of velocities fell within a flattened sphere of dimensions $\pm 400^{\circ} / \mathrm{s}$ torsional, $\pm 800^{\circ} / \mathrm{s}$ vertical, and $\pm 800^{\circ} / \mathrm{s}$ horizontal (Fig. $9 A$ ).

After unilateral riMLF inactivation, there was a stereotyped conjugate reduction in the quick phase velocity range of both animals. Eye velocities on one torsional side of List-

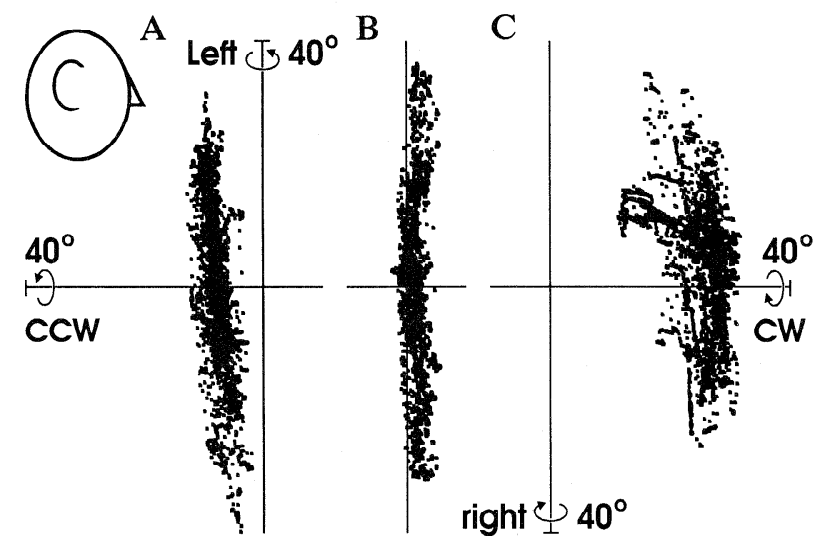

FIG. 10. Torsional shifting of the normal planar range of eye positions after unilateral rostral interstitial nucleus of the medial longitudinal fasciculus (riMLF) inactivation. Eye position quaternions from $100 \mathrm{~s}$ of visually guided, head-fixed saccades are plotted normally $(B)$, after right riMLF inactivation $(A)$, and after left riMLF inactivation $(C)$. Data are plotted in Listing's coordinates and viewed from the right side perspective, so that normal eye positions are confined to the vertical-horizontal plane perpendicular to the page. Data points are the tips of quaternion vectors that give the axis and magnitude of rotation from primary position, following the right-hand rule. Animal: $A R T$.

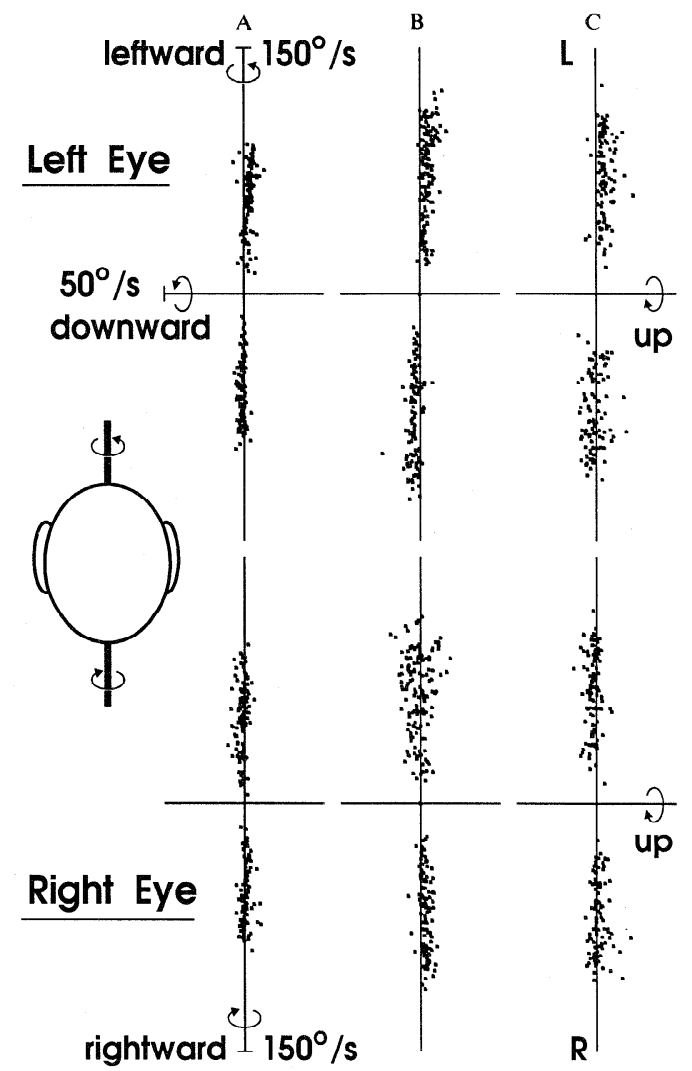

FIG. 11. Absence of abnormalities in horizontal slow-phase axes during unilateral rostral interstitial nucleus of the medial longitudinal fasciculus (riMLF) inactivation A: Normal. B: Left riMLF inactivation. C: Right riMLF inactivation. The postinjection data were measured in animal $A R T$ at the time of the torsional eye position shifts illustrated in Fig. 10, and the saccade axis tilts illustrated in Fig. 9. Normal tilts in slow-phase axes of the left eye and right eye were symmetrical across the midsaggital plane.

ing's plane was practically oblitcrated, the $\mathrm{CW}$ side during right riMLF inactivation (Fig. $9 \mathrm{~B}$, above views), and the CCW side obliterated during left inactivation (Fig. $9 C$, above view). The upward and downward range of velocities were equally reduced, usually by slightly more than $50 \%$ (Fig. 9, $B$ and $C$, both views), whereas the horizontal range was only slightly reduced (Fig. 9, $B$ and $C$, behind views). The remaining instantaneous eye velocity vectors formed an overall $3-\mathrm{D}$ range resembling a $90^{\circ}$ wedge cut out of the normal spherical range. These wedges fanned out torsionally from an inner narrow edge aligned with the vertical axis (Fig. 9, $B$ and $C$, above views) and were elongated along this axis (Fig. 9, $B$ and $C$, behind views). From the behind perspective, these wedges had a tilt that ranged from small (Fig. 9B) to quite prominent (Fig. 9C). Horizontal and vertical velocity appeared to be impaired more for oblique movements along one diagonal than along the other, an effect that was also consistently observed during trained oblique saccades. Velocities of both eyes are shown during right riMLF inactivation to illustrate the essentially conjugate nature of the deficit. The same basic deficit was observed in the velocities of head-fixed spontaneous saccades during all riMLF inactivations in all four animals, the main difference being that both the normal and abnormal torsional ranges were smaller in the absence of a vestibular stimulus. 
INTERACTIONS BETWEEN EYE VELOCITY AND POSITION. As expected from the torsional velocity imbalance described above, vertical saccades always drove eye position out of Listing's plane immediately after unilateral riMLF inactivation. These torsionally deviated positions were then held, unless the muscimol injection was placed at the border of INC (Crawford et al. 1991). As a result, the overall distribution of eye positions appeared to shift torsionally from the normal Listing's plane (Fig. $10 \mathrm{~B}$ ), CCW during right inactivation (Fig. 10 $A$ ) and $C W$ during left inactivation (Fig. $10 C)$. The position range shifted rapidly at first and then settled after 15-20 min, with a final mean torsional shift of $10-25^{\circ}$.

Was the linkage between horizontal and vertical saccade velocities (Figs. 8, $B$ and $C$, and $9, B$ and $C$, behind views ) a mechanical effect of the concurrent torsional eye positions? We hypothesized that, during tonic torsional deviations, the horizontal recti might take on elevator and depressor properties. To test this possibility, horizontal VOR slowphase axes were recorded during unilateral riMLF inactivation in animals $C A S$ and $A R T$. If there was an effect due to muscle mechanics, then slow-phase axes should tilt like the saccade axes. This was not the case (Fig. 11). Vertical axes of horizontal slow phases were not affected by the torsional eye positions produced by unilateral riMLF inactivation.

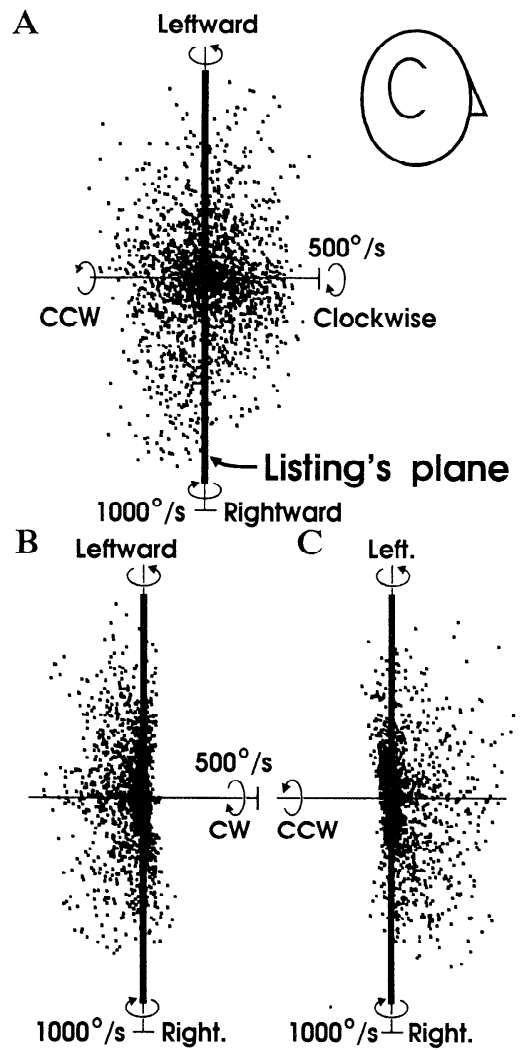

FIG. 12. Correspondence of saccade axes with Listing's plane after rostral interstitial nucleus of the medial longitudinal fasciculus ( riMLF) inactivation. Instantaneous eye velocities are plotted during normal visually guided torsional quick phases $(A)$, after right riMLF inactivation $(B)$, and after left riMLF inactivation $(C)$. Data are plotted in Listing's coordinates, i.e., Listing's plane is orthogonal to the page from the side view (thick line). Axes are largely confined behind Listing's plane during right riMLF inactivation $(B)$ and in front of Listing's plane during left inactivation $(C)$, i.e., Listing's plane aligned with the inner vertical edge of the intact wedge of velocities seen in Fig. 9. Animal: $A R T$.
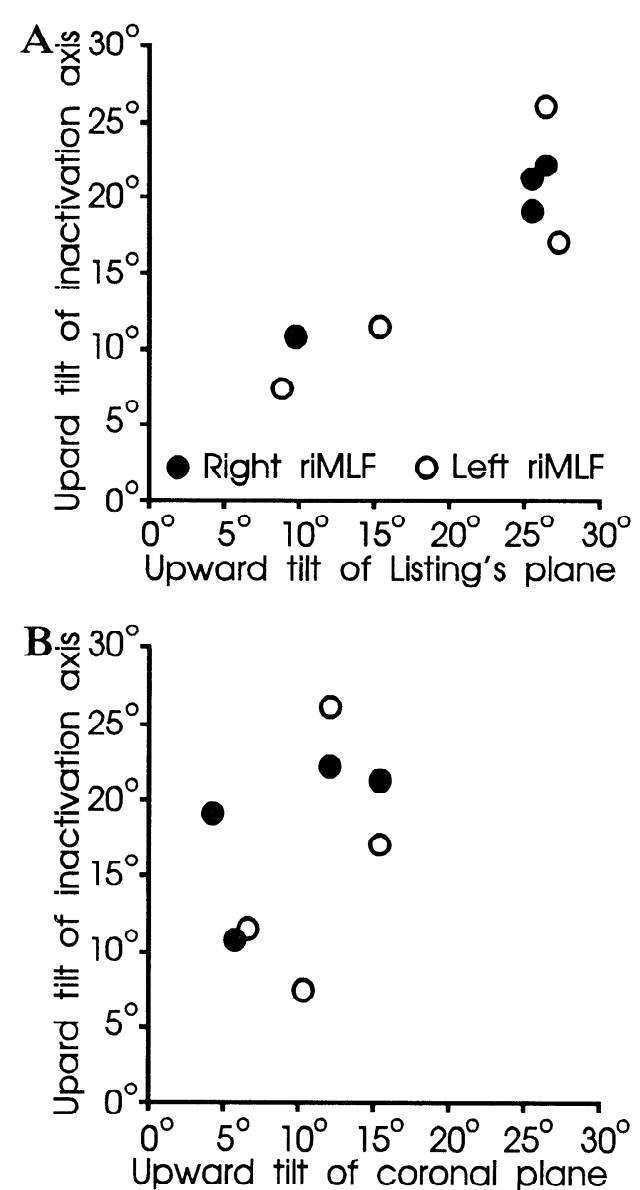

FIG. 13. Plot of upward tilts of axes fit to inactivation data (Fig. 12) as a function of upward tilt of Listing's planes $(A)$ and stereotaxic planes $(B)$ of all 4 animals, relative to magnetic field directions.

ALIGNMENT WITH IISTING'S PLANE. During unilateral inactivation, Listing's plane appeared to demarcate the division between the range of intact and missing torsional axes. This is most clear when viewing instantaneous eye velocities from the side, in Listing's coordinates (Fig. 12). Thus the deficits produced by injection of muscimol into one side compared with the other were symmetrical across Listing's plane, and the inner edge of the remaining velocity range is aligned closely with this plane.

To quantify this alignment in all animals, an axis was fit to the inner edge of the velocity range. A computer algorithm rotated a vertical axis about the origin to the orientation that maximized the number of velocity points on the intact side. Each computation was performed in field coordinates with the use of instantaneous velocities of $\sim 500$ saccades recorded over $100 \mathrm{~s}$. Collection of this data was initiated at $31 \pm 5$ (SE) min after injection of muscimol into the optimal riMLF sites discussed above. As done for the stimulation data, the upward tilts of these axes were then plotted against the upward tilts of Listing's plane (Fig. $13 \mathrm{~A}$ ), and the upward tilts of the stereotaxic planes (Fig. $13 \mathrm{~B}$ ) relative to field coordinates. Again, the relationship between the anatomic landmarks and the measured axis tilts was poor $\left(r^{2}=0.18\right.$; Fig. $\left.13 B\right)$, worse than the relationship between the anatomic landmarks and Listing's plane $\left(r^{2}=0.24\right.$; not illustrated). Again, the correlation between tilts of the inactivation-fit axes and Listing's plane was high 


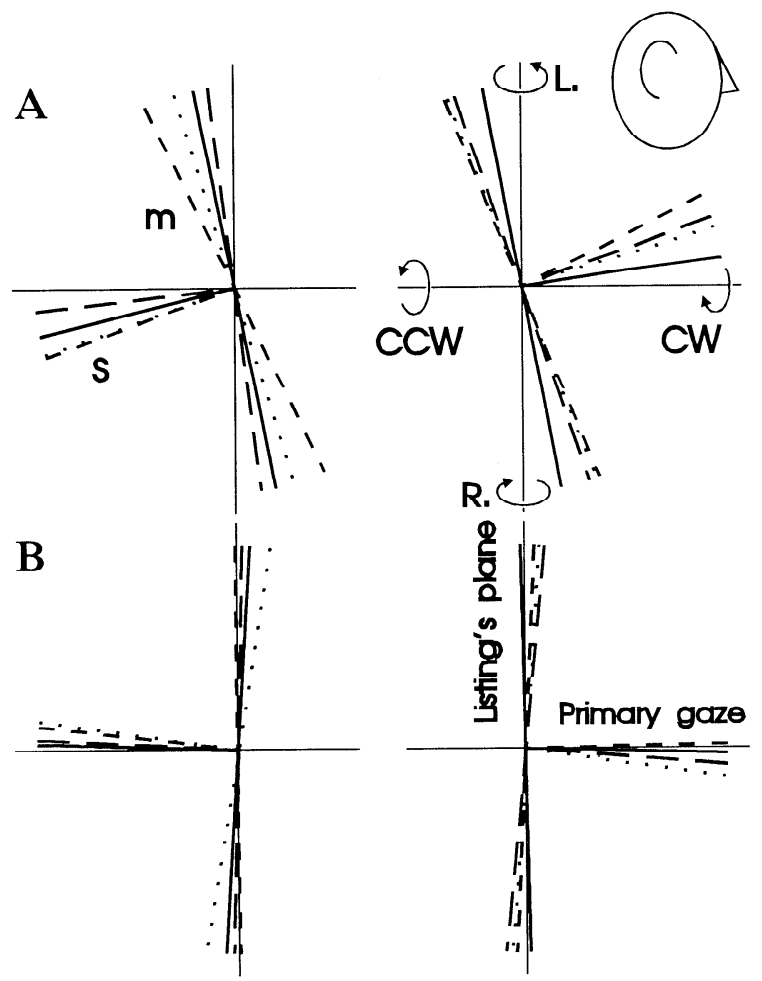

FIG. 14. Summary of alignment between rostral interstitial nucleus of the medial longitudinal fasciculus (riMLF) data and Listing's coordinates. Representative torsional stimulation axes (s) and vertical axes fit to unilateral inactivation data $(\mathrm{m})$ of optimal left riMLF (left column) and right riMLF (right column) sites of all 4 animals are shown. Data were computed in field coordinates $(A)$ and then rotated into Listing's coordinates $(B)$. Each animal's data are assigned a consistent line style throughout.

$\left(r^{2}=0.80\right)$, and the tilts of riMLF data and Listing's plane were approximately equal (Fig. $13 \mathrm{~A}$ ).

Alignment of riMLF stimulation and inactivation axes with Listing's coordinates is summarized in Fig. 14. Axes from the optimal riMLF sites of all animals (Figs. 4, 6, and 13 ) are viewed from the side. First, data are plotted in the field coordinate system in which the head was initially placed (Fig. 14A). This illustrates the variable upward tilts of the data in field coordinates, $16.2 \pm 6.3^{\circ}$ for stimulation data and $17.0 \pm 6.1^{\circ}$ for the inactivation data (mean \pm sample SD). Transformation into Listing's coordinates aligned the data with the coordinate axes and removed most of its variability (Fig. 14B). Stimulation axes now tilted downward by $4.0 \pm 3.1^{\circ}$, and inactivation axes tilted downward by $3.3 \pm 3.2^{\circ}$. The similarity of these two numbers in either coordinate system was related to the mutual orthogonality of the stimulation and inactivation data, i.e., $88.7 \pm 3.4^{\circ}$ apart for the left riMLF and $89.8 \pm 3.4^{\circ}$ for the right riMLF. Furthermore, in Listing's coordinates (Fig. $14 B$ ) it is clear that data from the left riMLF (left column) and right riMLF (right column) were nearly collinear; axes produced by left riMLF and right stimulation were $1.7 \pm$ $2.9^{\circ}$ apart, and axes fit to eye velocities during left and right riMLF inactivation were $4.7 \pm 0.7^{\circ}$ apart. Thus the data formed an orthogonal system that was aligned between the two sides of the brain, and furthermore, aligned with Listing's coordinates.

DISCUSSION

The oculomotor system appears to be an example of a neural network that expresses movement kinematics explic- itly and does so in a relatively clear hierarchy. Before considcring burst ncuron coordinates, it was necessary to corroborate several assumptions concerning the riMLFs position in this hierarchy. First, short-lead burst neuron firing rate appears to be related to eye velocity (King and Fuchs 1979; Van Gisbergen et al. 1981). If this relationship is causal, it follows that a constant stimulus input to burst neurons should produce a ramplike change in eye position. This was indeed observed during riMLF microstimulation. Second, because burst neurons are not active during fixation, their direct projections to motoneurons cannot hold eye position. Therefore it is thought that burst ncurons have an indirect path through a neural velocity-to-position transformation, which maintains motoneuron activity between saccades (Robinson 1975). This accounts for the observation that eye position held after each riMLF stimulation, as was observed for the PPRF (Cohen and Komatsuzaki 1972). Finally, injection of muscimol into the riMLF did not reveal any oculomotor deficits that could not be accounted for by burst neuron inactivation. The torsional deviations in eye positions observed during unilateral riMLF inactivation were the expected geometric consequence of a more fundamental deficit in saccade velocities, rather than positional drift. This supports the suggestion of single-unit recordings that this structure is homogenous in function, at least with respect to eye movements (Büttner et al. 1977; King and Fuchs 1979).

\section{Axes of eye rotation generated by riMLF burst neuron populations}

To specify the angular velocity of a rigid body such as the eye, at least three coordinate axes are required. Thus, in an orthogonal system, there must be at least six classes of excitatory short-lead burst neurons, i.e., three opponent pairs such as leftward-rightward, upward-downward, and CW$\mathrm{CCW}$. Although the techniques available (single-unit recording, electrical microstimulation, and injection of inhibitory drugs) each have their well-known limitations, together they provide strong evidence for six characterizable burst neuron populations. Burst neurons in the pontine PPRF mainly produce the horizontal component of saccades, each side containing a population that produces ipsilateral rotation (Cohen et al. 1968; Cohen and Komatsuzaki 1972). The present study confirms that each side of the riMLF contains two intermingled populations that produce upward and downward rotation (King and Fuchs 1979), the difference between the two sides being the effect on torsional rotation (Vilis et al. 1989).

Previous 3-D unit recording and riMLF inactivation studies (Henn et al. 1988; Hepp et al. 1989; Vilis et al. 1989) suggested that the four riMLF burst populations rotate the eye in directions similar to those that maximally activate the ipsilateral vertical semicircular canals (Blanks et al. 1985 ), i.e., CW-upward/CW-downward on the right side and $\mathrm{CCW}$-upward/CCW-downward on the left. This organization also resembles the pulling directions of the vertical muscles controlled by the ipsilateral motoneurons, in agreement with the primarily ipsilateral motoneuron projection sites of downward-tuned riMLF burst neurons (Moschovakis et al. 1991b; Simpson et al. 1986). However, recent anatomic evidence suggested that upward-tuncd burst neurons project to upward-tuned motoneurons on 
both sides of the brain (Moschovakis et al. 1991a), which would presumably cause their torsional components to cancel and result in purely upward rotation. According to this scheme, upward and CW-downward rotations would be encoded by the two right riMLF populations, whereas upward and CCW-downward would be encoded on the left. This assumes that the synaptic efficacies of burst neuron projections are all functionally equal, but this need not be the case. The presence of an anatomic connection says little about its functional efficacy. For example, vestibular neurons from each semicircular canal have an anatomic input to the motoneurons of every extraocular muscle, but only a minority provide a strong functional drive. There is reason to suspect that the contralateral synapses of upward burst neurons are not as efficacious as the ipsilateral synapses. For cxample, if these efficacies were equal and the upward neurons did not produce torsion, then downward-torsional burst neurons would have to be coactivated to a very high degree to produce upward-torsional rotations, which is not compatible with the cell recording literature (Vilis et al. 1989).

Our riMLF stimulations (CW for right riMLF, CCW for left ) were consistent with both the behaviorally and anatomically determined schemes. However, contrary to the anatomically determined scheme of Moschovakis et al., there did not appcar to be populations of burst neurons encoding purely upward saccades. When animals attempted to saccade between vertically displaced targets during unilateral inactivation, they could not produce vertical eye velocities without equal amounts of torsion (and some horizontal rotation). This suggests that the contralateral excitatory connections of riMLF burst neuron axons observed by Moschovakis et al. (1991a) have low synaptic weightings. If we assume that animals solely used either the up- or downrelated burst population of the intact riMLF side when saccading between vertically displaced targets, the four riMLF burst populations appeared to generate the following rotations: CW-up-left and CW-down-right in the right riMLF, and CCW-up-right and CCW-down-left in the left riMLF.
This proposed organization of riMLF burst neuron populations is illustrated schematically in Fig. 15, along with the previously described PPRF populations (Cohen et al. 1968; Cohen and Komatsuzaki 1972; Van Gisbergen et al. 1981). Because the activity of some PPRF burst neurons correlates best with oblique or even near-vertical directions (Keller 1974; Luschei and Fuchs 1972; Strassman et al. 1986), the characterization of these populations as purely horizontal may well be an oversimplification. Figure 15 also indicates which of these populations should be activated during clockwise $(A)$, upward $(B)$, and leftward $(C)$ rapid eye movements. To illustrate how this scheme fits with the data, imagine that an upward saccade is attempted in the absence of the right riMLF, assuming normal ratios of input to the burst neuron populations. The eyes should rotate in the upward-CCW-rightward direction controlled by the population on the intact side, as observed experimentally. This scheme provides a unifying interpretation for the results in this investigation.

The above organization surprisingly suggests that riMLF burst neurons might contribute to normal horizontal rotations, which were previously thought to be generated exclusively by PPRF burst neurons. If so, then riMLF inactivation should reduce horizontal saccade velocity (although not to the extent of torsional and vertical velocity ). Furthermore, riMLF populations would have to be bilaterally activated during normal horizontal saccades to cancel out their major torsional and vertical components, and these components should no longer cancel during unilateral inactivation. For example, if a leftward saccade is attempted in the absence of the right riMLF, Fig. $15 C$ predicts that the downward component produced by the opposite intact side will no longer be cancelled. The predicted impairment of horizontal velocity and patterns of inappropriate vertical rotations were indeed observed. Initially, we thought these abnormal axis tilts might be a mechanical product of the abnormal torsional eye positions that existed concurrently. Muscle pulling directions appear to be relatively independent of two-dimensional (2-D) eye positions (Miller and

\section{A Clockwise Rotation}
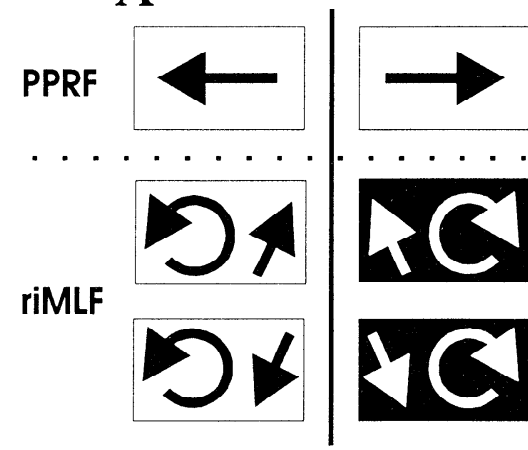

B Upward Rotation
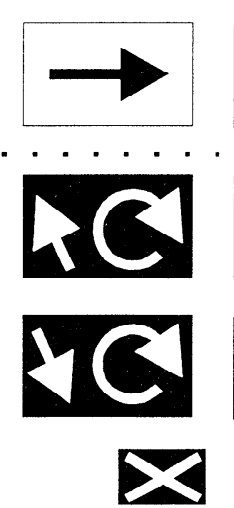

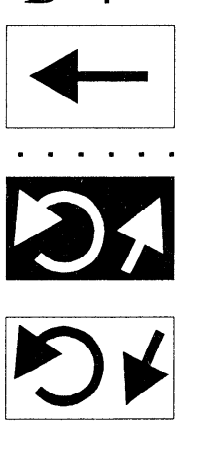

active

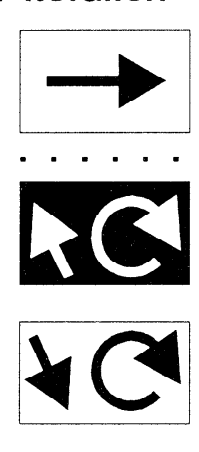

$\times$ inactive
C Leftward Rotation
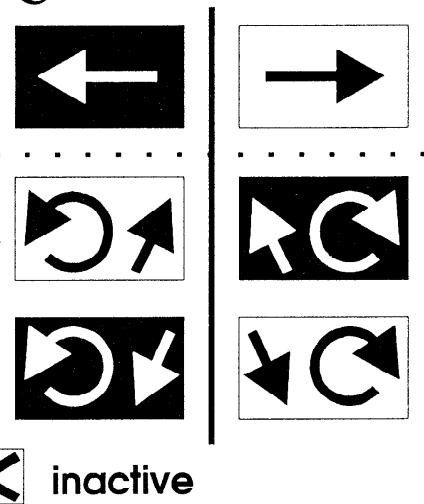

FIG. 15. Experimentally determined scheme for directions of eye rotation generated by the 6 known excitatory short-lead burst neuron populations, showing the patterns of activation that should occur during torsional $(A)$, vertical $(B)$, and horizontal $(C)$ rapid eye movements. Each rectangular box represents 1 burst neuron population, illustrated in symmetry across the midsagittal plane (vertical lines). Arrows indicate the direction of eye movement produced by the population. Dotted lines divide between the top 2 paramedian pontine reticular formation populations and the bottom 4 rostral interstitial nucleus of the medial longitudinal fasciculus ( riMLF) populations. Activation is defined as greater activity relative to the inactive populations. Single-unit recordings suggest that riMLF neurons are activated more for torsional $(A)$ and vertical $(B)$ rotations than for comparably sized horizontal $(C)$ rotations (Büttner et al. 1977; Hepp et al. 1988 ). 
Robins 1987), but torsional dependence has not been examined. However, VOR slow-phase axes did not exhibit the tilts observed in saccade axes. Barring the unlikely possibility that the VOR makes position compensations that the saccadic system does not, eye muscle pulling directions do not appear to be dependent on the torsional deviations produced by unilateral riMLF inactivation.

Is involvement of riMLF burst ncurons in generating horizontal saccades consistent with previous literature? Early 2-D single-unit recordings of riMLF burst neurons suggested oblique tilts in directional tuning that agree with our data (Büttner et al. 1977), and anatomic studies have identified projections from the riMLF to the abducens nucleus that were previously difficult to explain (Büttner-Ennever and Büttner 1988). Our observation appears to contradict the finding that PPRF lesions can completely obliterate horizontal saccades (Cohen et al. 1968; Henn et al. 1984), but interpretation of this is problematic. The PPRF possesses short- and long-lead burst neurons (Raybourn and Keller 1977), and the latter are thought to project to the riMLF (Büttner-Ennever and Büttner 1978, 1988). Thus PPRF inactivation might remove the inputs that normally activate riMLF burst neurons during horizontal saccades.

To summarize how the Fig. 15 scheme accounts for the remaining results, first, stimulation of the two (intermingled) riMLF populations on one side of the brain should result in torsional eye rotation, whereas vertical and horizontal components cancel (as in Fig. 15A). Second, the linkage between horizontal and vertical rotation in riMLF burst populations accounts for the greater difficulty in saccading along one oblique diagonal than the other during unilateral riMLF inactivation. Finally, if one side of the schematic riMLF is eliminated, the set of all possible combinations of the remaining directions resembles the full range of velocities that we observed after unilateral riMLF inactivation. Thus the distribution of intact eye velocities during visually guided torsional quick phases tilted obliquely and showed a reduction in vertical velocity by at least $50 \%$ and a total absence of rotation in one torsional direction.

Why is it that burst neurons are basically organized into a separate horizontal saccade generator, and a combined vertical-torsional generator in the midbrain? Because these directions are similarly combined in the eye muscles (Simpson et al. 1986), the semicircular canals (Blanks et al. 1985), and the oculomotor integrator (Crawford et al. 1991), it would appear that there is something fundamental about orienting rotations of the eye and head that makes this organization optimal. By post-hoc arguments, it can be shown that this unique near-orthogonal arrangement optimizes several important principles, including signal-tonoise ratio, bilateral symmetry, nonredundancy, and energy efficiency (Crawford and Vilis 1991; Robinson 1985; Simpson and Graf 1985). Figure 16 illustrates and explains the basic arguments. Figure $16 E$ also shows that the measured riMLF axes deviate slightly from the ideal orthogonal system in Fig. $16 D$, as indicated by the horizontal tilts in the vertical arrows. These additional horizontal signals appear to satisfy bilateral symmetry but introduce nonorthogonality and redundancy.

Why would the saccadic system sacrifice orthogonality to introduce signals that are apparently redundant? Thus far, our theoretical discussions have mainly considered orthogo-
A

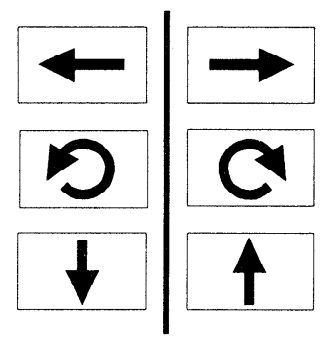

B

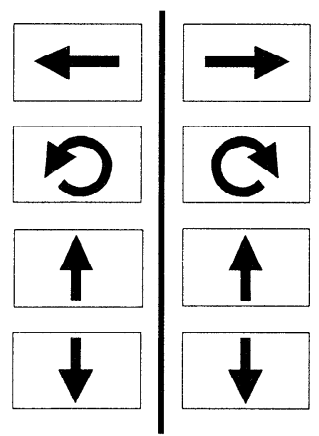

C

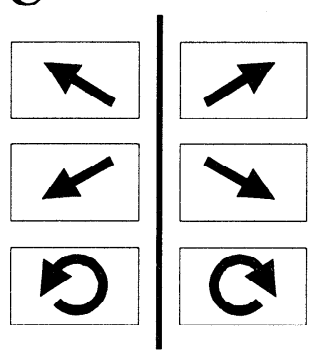

D

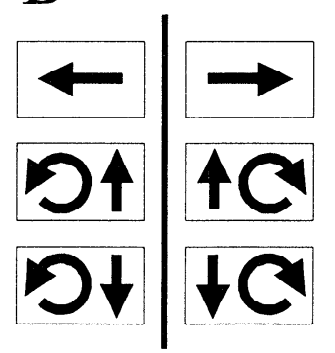

E

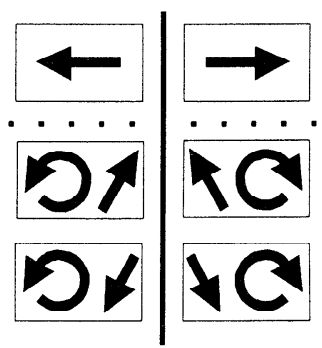

FIG. 16. Hypothetical schemes for dividing directions of rotation among the burst neuron populations. Burst neuron populations are represented as in Fig. 17. It is assumed that orthogonal coordinates are optimal because they enhance the signal to noise ratio, i.e., the angle between any 2 coordinate axes in such a system is large compared with the biological noise in the individual channels (Robinson 1985). A Cartesian scheme $(A)$ would divide the vestibular-oculomotor systems into 3 structures for torsional, vertical, and horizontal rotations, with 1 direction of each rotation represented per side, e.g., clockwise on the right and counterclockwise on the left brain. The problem with this representation is that up and down are not mirror symmetrical between the 2 sides of the head across the midsaggital plane (Robinson 1985). With a Cartesian system the neurons of the right brain would have to make anatomically asymmetric connections to sensory and motor structures compared with the left brain. To maintain symmetry, up and down have to be equally represented on each side of the brain, but the cost of simple duplication $(B)$ is redundancy (Simpson and Graf 1985). Another way to achieve symmetry is to combine vertical with one of the other directions, either horizontal $(C)$, or torsional $(D)$. Which choice is optimal? Terrestrial animals live in a primarily horizontal environment defined by the earth's surface. Thus they make primarily horizontal orienting movements, whereas torsional movements are minimized. The horizontal-vertical combination $\left(C^{\prime}\right)$ requires coactivation of 2 oblique eye muscles during every horizontal rotation. Such coactivation is useful for stabilization of joints against unpredictable loads but would be a waste of energy for the relatively constant oculomotor plant. The torsional-vertical system $(D)$, which resembles eye muscles and canals, would not require coactivation during horizontal rotations and so has the advantage of energy efficiency. Our experimentally determined scheme for the rostral interstitial nucleus of the medial longitudinal fasciculus $(E)$ is similar but has additional horizontal signals that introduce redundancy and nonorthogonality. 
nal burst neuron coordinate systems, which require three opponent pairs of populations encoding opposite directions of rotation about the same axis. By encoding rotations about four different axes in the experimentally measured arrangement, the riMLF gains the potential to generate eye rotations about any 3-D axis. For example, by picking various combinations of two out of the four riMLF populations, purely torsional, vertical, or horizontal rotations could be made (consider Fig. 15, in absence of the PPRF populations). These results are an illustration of a theorctical possibility that has previously been ignored by neuroscientists, determination of 3-D rotations with a minimum of four neuron populations. This might explain how some aquatic organisms achieve a 3-D VOR with only four semicircular canals (Rovainen 1976). Because 2-D experiments suggest that the PPRF may control horizontal and vertical rotations and this structure has not been explored three-dimensionally, it is possible that this nucleus has a similar 3-D arrangement, but with more emphasis on horizontal. Thus it appears that motor control systems may use simple 3-D coordinate systems embedded within more complex overall coordinate systems, although the functional utility of this is not presently clear.

\section{Alignment with Listing's plane}

It is clear that motoneuron and sensory afferent coordinates are determined by the anatomic structures they innervate, but it is less clear what coordinate systems are used by the intervening structures. Previous thcorctical and experimental investigations of motor coordinates have argued between various systems on the basis of anatomic parameters, including muscle pulling directions (Büttner et al. 1977; Pellionisz 1986), other parameters of the physical plant (Hollerbach and Atkeson 1987; Soechting and Flanders 1991), sensory anatomy (Robinson and Zee 1981), or intermediate stages between sensory and motor structures (Peterson and Baker 1991). However, we found that the orientation of rotation axes controlled by burst neuron populations did not correlate well with externally measured anatomic landmarks. Furthermore, when the full compliment of torsional, vertical, and horizontal elements of riMLF burst population coordinates are considered, they did not resemble either vertical eye muscles or vertical canals as much as previously expected (Büttner et al. 1977; Robinson and Zee 1981).

Another possibility is that motor systems sometimes utilize coordinates that align with some property of behavior such as Listing's plane of saccadic eye positions, which varies with respect to anatomic landmarks from subject to subject (Tweed and Vilis 1990b). We found that burst neuron coordinates did correlate well with the orientation of Listing's plane. Clearly, the individual riMLF burst populations did not produce rotations about axes parallel or orthogonal to Listing's plane. However, both stimulation and inactivation cxperiments suggested that these axes are symmetrical across Listing's plane, and about the primary gaze direction orthogonal to Listing's plane. During unilateral riMLF stimulation the vertical and horizontal components of two populations appeared to cancel, leaving a torsional axis of rotation orthogonal to Listing's plane, i.e., parallel to the primary gaze direction. Normally, rapid eye movement axes are not confined to Listing's plane (Tweed and Vilis 1990b). After riMLF inactivation, horizontal saccade axes, presumably produced primarily by the PPRF, appeared to align with Listing's plane. These stimulation and inactivation axes were mutually orthogonal, aligned well between the two sides of the brain, and collapsed along the coordinate axes when transformed into Listing's coordinates.

Figure 17 summarizes the axes of eye rotation controlled by the six known burst neuron populations, plotted symmetrically about Listing's coordinates. The PPRF appears to mainly rotate the eye about the vertical axis in Listing's plane. The mainly torsional and vertical role of the left riMLF and right riMLF populations is best seen from the above view (Fig. 17 A), whereas the other views also show the smaller horizontal component. Although the illustrated riMLF axes do not align with Listing's plane (L.P.) or the primary gaze direction $(\mathrm{P})$, they are symmetrical about these coordinates.

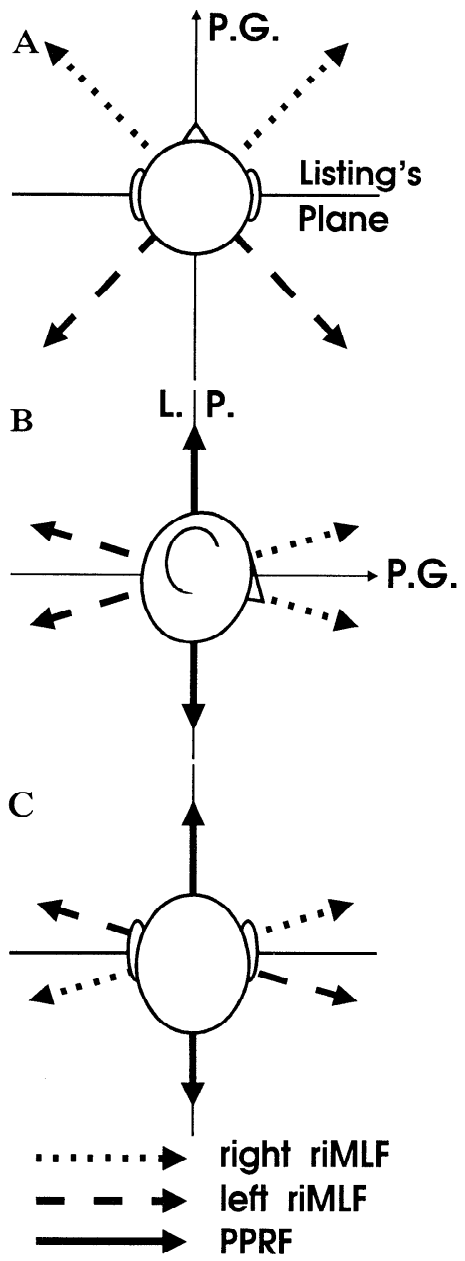

FIG. 17. Axes of eye rotation controlled by the various short-lead burst neuron populations. Axes based on experimental data are plotted in Listing's coordinates and viewed from above Listing's plane with the primary gaze direction (P.G.) pointing up on the page $(A)$, the side of Listing's plane (L.P.) with P.G. pointing rightward $(B)$, and behind Listing's plane (C). Note that these axes define a coordinate system that is symmetrical about Listing's coordinates but do not all align with Listing's plane or the primary gaze direction. The paramedian pontine reticular formation is treated as having only 2 distinguishable populations, although this may be an oversimplification. Directions of rotation are defined by the right-hand rule. 
Had this data aligned with either canal or muscle coordinates, one would expect that the stimulation axes would lay in the plane of the horizontal canals/muscles with the inactivation axes extending orthogonally. When our data (Fig. 14 ) were replotted into coordinate systems based on anatomic data measured previously in rhesus monkeys, the stimulation/inactivation data tilted by $15.7 \pm 6.3^{\circ} / 14.9 \pm$ $5.7^{\circ}$ downward from canal coordinates (Blanks et al. 1985) and $8.6 \pm 6.3^{\circ} / 7.8 \pm 5.7^{\circ}$ downward from muscle coordinates (Simpson et al. 1986), compared with $4.0 \pm 3.1^{\circ}$ \% $3.3 \pm 3.2^{\circ}$ from Listing's coordinates (mean \pm sample $\mathrm{SD}$ ). Thus, of these two anatomic schemes, muscle coordinates on average aligned better with the riMLF data and Listing's plane. Because we did not directly measure muscle pulling directions, we cannot eliminate the possibility that they also varied with respect to stereotaxic coordinates as much as Listing's plane. However, the Listing's coordinates we measured varied with respect to stereotaxic coordinates with a standard deviation of $\pm 6.9^{\circ}$. This appears to be much larger than the variations of muscle coordinates reported among three cats (Ezure and Graf 1984), but a study of variations between a larger population of primates would be welcome.

Does alignment of burst neuron coordinates with Listing's plane make sense? Burst neurons appear to be dedicated primarily to saccade production. The saccadic system has three degrees of freedom with which to perform the 2-D task of foveating targets. Listing's law appears to be the brain's solution to this kinematic redundancy. To have such a behavioral constraint, there must be a constraint on neural activity (Crawford and Vilis 1991). Given any one coordinate system, it is Listing's law that determines the axis of rotation for a given saccadic gaze shift, and thus the extent to which each burst population is activated. Conversely, given Listing's law, the coordinate system used will determine the extent to which each population is activated. Thus it is not so surprising that there is a correspondence between these two interrelated factors.

It follows that wherever constraints on motor behavior are observed, they may have correlates in both neural activity and coordinate systems. The smooth pursuit system, which has the same kinematic redundancy as the saccadic system, is constrained even more closely by Listing's law (Tweed et al. 1992) and so might be expected to utilize a Listing-based coordinate system. In contrast, optimal vestibuloocular and optokinetic responses are essentially dictated by a 3-D sensory input (assuming their function is to stabilize retinal slip) and do not require additional constraints (Crawford and Vilis 1991). As might be expected, their interneurons appear to utilize coordinates similar to those of the semicircular canals, eye muscles, or some combination of these (Baker et al. 1984; Peterson and Baker 1991; Simpson et al. 1989). Suprisingly, VOR coordinates may not be completely independent of Listing's law, because the axis of minimal VOR gain appeared to align with the primary gaze direction in the monkey (Crawford and Vilis 1991). Such coordinate interdependence might result from sharing of circuits between various oculomotor systems, for example at the oculomotor integrator.

What then of skeletomotor systems whose circuits generate multiple, complex behaviors? Recently, it has been demonstrated that head and arm orientations are constrained to two dimensions during looking and pointing by a rule similar to Listing's law (Glenn and Vilis 1992; Hore et al. 1992; Straumann et al. 1991). Could these constraints have correlates in neural coordinate systems, and, if so, how do they interact with the coordinates used for other behavioral constraints? The oculomotor system, with only two basic 3-D strategies (Listing's law and retinal stabilization ) provides a valuable model for such questions.

We thank L. Van Clecf and S. Watts for technical assistance. Wc also thank J. Hore and D. Tweed for editorial comments.

This study was supported by Medical Research Council Grant MT9335. During the period of this study, J. D. Crawford held a Medical Research Council Studentship.

Address for reprint requests: T. Vilis, Dept. of Physiology, University of Western Ontario, London, Ontario N6A 5C1, Canada.

Received 26 August 1991; accepted in final form 9 April 1992.

\section{REFERENCES}

ANASTASIO, T. J. AND RoBinson, D. A. Distributed parallel processing in the vertical vestibuloocular reflex: learning networks compared to tensor theory. Biol. Cyhern. 63: 161-167, 1990.

ANDERson, R. A., EssiK, G. K., AND ZIPSER, D. Encoding of spatial location by posterior parietal neurons. Science Wash. DC 230: 456-458, 1985.

Baker, J., Goldgerg, J., Hermann, G., and Peterson, B. Optimal response planes and canal convergence in secondary neurons in vestibular nuclei of alert cats. Brain Res. 294: 133-137, 1984.

Blanks, R. H. I., Curthoys, I. S., BenNet, M. L., ANd Markham, C. H. Planar relationships of the semicircular canals in rhesus and squirrel monkeys. Brain Res. 340: 315-324, 1985.

BüTTNER, U., BÜTTNER-ENNEVER, J. A., AND HENN, V. Vertical eye unit related activity in the rostral mesencephalic reticular formation of the alert monkey. Brain Res. 130: 239-252, 1977.

BüTTNER-ENNEVER, J. A. AND BÜTTNFR, U. A cell group associated with vertical eye movements in the rostral mesencephalic reticular formation of the monkey. Brain Res. 151: 31-47, 1978.

BÜTTNER-ENNEVER, J. A. AND BÜTTNER, U. The reticular formation. In: Neuroanatomy of the Oculomotor System, edited by J. A. BüttnerEnnever. Amsterdam: Elsevier, 1988, p. 135-136, 148-149.

CoHEN, B. AND KomatsuzaKi, $\Lambda$. Eye movements induced by stimulation of the pontine reticular formation: evidence for integration in oculomotor pathways. Exp. Neurol. 36: 101-117, 1972

COHEN, B., Komatsuzaki, A., AND Bender, M. B. Electrooculographic syndrome in monkeys after pontine reticular formation lesions. Arch. Neurol. 18: 78-92, 1968.

Crawford, J. D., CAdera, W., ANd Vilis, T. Generation of torsional and vertical eye position signals by the interstitial nucleus of Cajal. Science Wash. DC 252: 1551-1553, 1991.

CRAWFORD, J. D. AND VILIS, T. Axes of eye rotation and Listing's law during rotations of the head. J. Neurophysiol. 65: 407-423, 1991.

EzURE, K. AND GRAF, W. A quantitative analysis of the spatial organization of the vestibulo-ocular reflexes in lateral- and frontal-eyed animals. I. Orientation of the semicircular canals and extraocular muscles. Neuroscience 12: 85-93, 1984.

Ferman, L., Collewijn, H., ANd Van Den Berg, A. V. A direct test of Listing's law. II. Human ocular torsion measured under dynamic conditions. Vision Res. 27: 939-951, 1987.

FuchS, A. F., KANEKo, C. R. S., AND Scudder, C. A. Brainstem control of saccadic eye movements. Annu. Rev. Neurosci. 8: 307-337, 1985.

Fukushima, K. The interstitial nucleus of Cajal and its role in control of movements of head and eyes. Prog. Neurobiol. 29: 107-192, 1987.

GLENN, B. AND VILIS, T. Violations of Listing's law following large eye and head gaze shifts. J. of Neurophysiol. 68: 309-318, 1992.

HASSLER, R. Supranuclear structures regulating binocular eye and head movements. In: Cerebral Control of Eye Movements and Motion Perception, Bibleothica Ophthalmologica, editcd by J. Dichgans and E. Bizzi. Basel: Karger, 1972, vol. 82, p. 207-219.

Von Helmholtz, H. Treatise on Physiological Optics (English Translation), translated by J. P. C. Southall. Rochester, NY: Opt. Soc. Am., 1925 , vol. 3 , p. $44-51$ 
HENN, V., HePP, K., AND VILIS, T. Rapid eye movement generation in the primate: physiology, pathophysiology, and clinical implications. Rev. Neurol. Paris 145: 540-545, 1989.

Henn, V., LANG, W., HePp, K., AND ReISINE, H. Experimental gaze palsies in monkeys and their relation to human pathology. Brain 107: 619636, 1984.

Hepp, K. On Listing's law. Commun. Math. Phys. 132: 285-292, 1990.

HEPP, K., VILIS, T., AND HENN, V. On the generation of rapid eye movements in three dimensions. In: Representation of Three-Dimensional Space in the Vestibular, Oculomotor and Visual Systems, edited by B. Cohen and V. Henn. New York: Ann. NY Acad. Sci., 1988, vol. 545, p. $140-153$.

Hollerbach, J. M. AND AtKeson, C. G. Deducing planning variables from experimental arm trajectories: pitfalls and possibilities. Biol. $\mathrm{Cy}$ bern. 56: 279-292, 1987.

Hore, J., WatTS, S., AND VILIS, T. Constraints on arm position when pointing in three dimensions: Donder's law and the Fick Gimbal Strategy. J. Neurophysiol. 68: 374-383, 1992.

KELLER, E. L. Participation of medial pontine reticular formation in eye movement generation in monkey. J. Neurophysiol. 37: 316-332, 1974.

KING, W. M. AND FUCHS, A. F. Reticular control of vertical saccadic eye movements by mesencephalic burst neurons. J. Neurophysiol. 42: 861876, 1979.

Kompf, D., Pasik, T., Pasik, P., and Bender, M. B. Downward gaze in monkcys. Stimulation and lesion studies. Brain 102: 527-558, 1979.

LUSCHEI, E. S. AND FUCHS, A. F. Activity of brain stem neurons during eye movements of alert monkeys. J. Neurophysiol. 35: 445-461, 1972.

MAYS, L. E. AND SPARKS, D. L. Saccades are spatially not retinocentrically encoded. Science Wash. DC 208: 1163-1165, 1980.

Miller, J. M. AND Robins, D. Extraocular muscle sideslip and orbital geometry in monkeys. Vision Res. 27: 381-392, 1987.

Moschovakis, A. K., Scudder, C. A., AND Highstein, S. M. Structure of the primate oculomotor burst generator. I. Medium lead burst neurons with upward on-directions. J. Neurophysiol. 65: 203-217, 1991a.

Moschovakis, A. K., SCUdDer, C. A., Highstein, S. M., AND WARren, J. D. Structure of the primate oculomotor burst generator. II. Medium lead burst neurons with downward on-directions. J. Neurophysiol. 65: 218-229, 1991 b.

PELLIONISZ, A. Tensorial relationship found for structural \& functional reference frames of brain function: saccade neurons in monkey utilize frames composed of the eigenvectors of the frame of oculomotor muscles. Soc. Neurosci. Abstr. 12: 1186, 1986.

Pellionisz, A. J. And Peterson, B. W. A tensorial model of neck motor activation. In: Control of Head Movement, edited by B. W. Peterson and F. J. Richmond. New York: Oxford Univ. Press, 1988, p. 178-186.

PeTERSON, B. W. AND BAKER, J. F. Spatial transformations in vestibular reflex systems. In: Motor Control: Concepts and Issues (Dahlem Workshop Reports), edited by D. R. Humphrey and H.-J. Freund. New York: Wiley, 1991, p. 121-135.

RAPHAN, T. AND COHEN, B. Multidimensional organization of the vestibulo-ocular reflex (VOR). In: Adaptive Processes in Visual and Oculomotor Systems, edited by E. L. Keller and D. S. Zee. Oxford, UK: Pergamon, 1986, p. 285-292.

RAYBOURN, M. S. AND KeLler, E. L. Colliculoreticular organization in primate oculomotor system. I. Neurophysiol. 40: 861-878, 1977.

RoBINson, D. A. Oculomotor control signals. In: Basic Mechanisms of Ocular Motility and Their Clinical Implications, edited by P. Bach-yRita and G. Lennerstrand. Oxford, UK: Pergamon, 1975, p. 337-374 (Wenner-Gren Cent. Int. Symp. Ser.).

RoBINSON, D. A. The coordinates of neurons in the vestibulo-ocular re- flex. In: Adaptive Mechanisms in Gaze Control. Facts and Theories, edited by A. Berthoz and G. Melvill Jones. Amsterdam: Elsevier, 1985, p. 297-311.

RoBInson, D. A. AND ZEE, D. S. Theoretical considerations of the function and circuitry of various rapid eye movements. In: Progress in $\mathrm{OCu}$ lomotor Research. Developmental Neuroscience, edited by A. F. Fuchs and W. Becker. New York: Elsevier/North-Holland, 1981, vol. 12, p. 3-9.

Rovainen, C. M. Vestibulo-ocular reflex in adult sea lampreys. J. Comp. Physiol. 112: 159-164, 1976.

SCUdDER, C. A., FuCHS, A. F., AND LANGER, T. P. Characteristics and functional identification of saccadic inhibitory burst neurons in the alert monkey. J. Neurophysiol. 59: 1430-1454, 1988.

Shanthı, T. R., Manocha, S. L., and Bourne, G. H. A Stereotaxic Atlas of the Java Monkey Brain. New York: Karger, 1968, p. 32.

SimPSON, J. I. AND GRAF, W. The selection of reference frames by nature and its investigators. In: Adaptive Mechanisms in Gaze Control. Facts and Theories, edited by A. Berthoz and G. Melvill Jones. Amsterdam: Elsevier, 1985, p. 3-16.

Simpson, J. I., Rudinger, D., Reisine, H., ANd Henn, V. Geometry of extraocular muscles of the rhesus monkey. Soc. Neurosci. Abstr. 12: $1186,1986$.

Simpson, J. I., VAN der Steen, J., TAN, J., Graf, W., and LeOnard, C. S. Representations of ocular rotations in the cerebellar flocculus of the rabbit. Prog. Brain. Res. 80: 213-223, 1989.

SOECHTING, J. F. AND FLANDERS, M. Deducing central algorithms of arm movement control from kinematics. In: Motor Control: Concepts and Issues (Dahlem Workshop Reports), edited by D. R. Humphrey and H.-J. Freund. New York: Wiley, 1991, p. 293-306.

SPARKS, D. L. AND MAYS, L. E. Signal transformations required for the generation of saccadic eye movements. Annu. Rev. Neurosci. 13: 309_ 336, 1990.

Strassman, A., Highstein, S. M., and McCrea, R. A. Anatomy and physiology of saccadic burst neurons in the alert squirrel monkey. I. excitatory burst ncurons. J. Comp. Neurol. 249: 337-357, 1986.

Straumann, D., Haslwanter, T., Hepp-Reymond, M. C., AND Hepp, $K$. Listing's law for the eye, head and arm movements and their synergistic control. Exp. Brain. Res. 86: 209-215, 1991.

TweED, D., CADERA, W., AND VILIS, T. Computing three dimensional eye position quaternions and eye velocity from search coil signals. Vision Res. 30: 97-110, 1990.

Tweed, D., Fetter, M., Andreadaki, S., Koenig, E., And Dichgans, J. Three-dimensional properties of pursuit eye movements. Vision Res. In press.

TweED, D. AND VILIS, T. The superior colliculus and spatiotemporal translation in the saccadic system. Neural Networks 3: 75-86, 1990a.

TWEED, D. AND VIIIS, T. Geometric relations of eye position and velocity vectors during saccades. Vision Res. 30: 111-127, 1990b.

VAN Gisbergen, J. A. M., Robinson, D. A., AND Gielen, S. A quantitative analysis of generation of saccadic eye movements by burst neurons. J. Neurophysiol. 45: 417-442, 1981.

Vilis, T., HePP, K., Schwarz, U., AND HenN, V. On the generation of vertical and torsional rapid eye movements in the monkey. Exp. Brain Res. 77: 1-11, 1989.

Westheimer, G. Kinematics of the eye. J. Opt. Soc. Am. 47: 967-974, 1957.

WeStheimer, G. AND Blair, S. M. Mapping the visual sensory onto the visual motor system. Invest. Ophthalmol. 11: 490-496, 1972.

WESTHEIMER, G. AND BLAIR, S. M. The ocular tilt reaction-a brainstem oculomotor routine. Invest. Ophthalmol. 14: 833-839, 1975. 